\title{
Galactic Structure in the Outer Disk: The Field in the Line of Sight to the Intermediate-Age open Cluster Tombaugh $1^{*}$
}

\author{
Giovanni Carraro ${ }^{1,5}$, Joao Victor Sales Silva ${ }^{2,5,6}$, Christian Moni Bidin $^{3}$, and Ruben A. Vazquez ${ }^{4}$ \\ ${ }^{1}$ Dipartimento di Fisica e Astronomia, Universitá di Padova Vicolo Osservatorio 3 I-35122, Padova, Italy; giovanni.carraro@unipd.it \\ 2 Observatorio Nacional/MCT Rua Gen. José Cristino 77 20291-400, Rio de Janeiro, Brazil \\ ${ }^{3}$ Instituto de Astronomia, Universidad Catolica del Norte Av. Angamos 0610, Casilla 1280 Antofagasta, Chile \\ ${ }^{4}$ Instituto de Astrofisica de La Plata CONICET/ UNLP, Paseo del Bosque s/n La Plata, Argentina \\ Received 2016 July 31; revised 2016 November 22; accepted 2016 December 12; published 2017 February 7
}

\begin{abstract}
We employ optical photometry and high-resolution spectroscopy to study a field toward the open cluster Tombaugh 1, where we identify a complex population mixture that we describe in terms of young and old Galactic thin disks. Of particular interest is the spatial distribution of the young population, which consists of dwarfs with spectral types as early as B6 and is distributed in a blue plume feature in the color-magnitude diagram. For the first time, we confirm spectroscopically that most of these stars are early-type stars and not blue stragglers or halo/ thick-disk subdwarfs. Moreover, they are not evenly distributed along the line of sight but crowd at heliocentric distances between 6.6 and $8.2 \mathrm{kpc}$. We compare these results with present-day understanding of the spiral structure of the Galaxy and suggest that they trace the outer arm. This range of distances challenges current Galactic models adopting a disk cutoff at $14 \mathrm{kpc}$ from the Galactic center. The young dwarfs overlap in space with an older component, which is identified as an old Galactic thin disk. Both young and old populations are confined in space since the disk is warped at the latitude and longitude of Tombaugh 1 . The main effects of the warp are that the line of sight intersects the disk and entirely crosses it at the outer arm distance and that there are no traces of the closer Perseus arm, which would then be either unimportant in this sector or located much closer to the formal Galactic plane. Finally, we analyze a group of giant stars, which turn out to be located at very different distances and to possess very different chemical properties, with no obvious relation to the other populations.
\end{abstract}

Key words: Galaxy: disk - Hertzsprung-Russell and C-M diagrams - open clusters and associations: general stars: abundances

\section{Introduction}

Color-magnitude diagrams (CMDs) of stellar fields in the third quadrant of the Milky Way have repeatedly unraveled the remarkable complexity of stellar populations in the outer Galactic disk. Beyond any doubt, two dominating features appear on top of the main sequence (MS) of the nearby Galactic field: a thick MS having a prominent turnoff (TO) point and made up of intermediate-age stars poor in metals and an almost vertical sequence of young blue stars, known as the blue plume (Carraro et al. 2005, 2016; Moitinho et al. 2006). This complexity was originally interpreted as the result of the accretion of a satellite onto the Milky Way disk (Bellazzini et al. 2004; Martin et al. 2004) and of different age and metallicity sequences described as different episodes of star formation in an individual stellar system, the Canis Major dwarf galaxy.

The blue plume deserves particular attention, since to date no general consensus exists about its nature. The reason is that in the vast majority of cases, only photometric data are available, which are not univocal enough to derive solid estimates of the stars' gravity and temperature and, in turn, to infer their spectral type and distance. Because of the partial leverage of photometric data, various interpretations of the blue plume are available. It can be made up of genuine young blue stars and describe either the last episode of star formation in Canis

\footnotetext{
* Based on observations carried out at Las Campanas Observatory, Chile (program ID CN009B-042), and Cerro Tololo Inter-American Observatory.

5 ESO, Alonso de Cordoba 3107, Santiago de Chile, Chile.

${ }^{6}$ Instituto de Astronomia, Universidad Catolica del Norte, Antofagasta, Chile.
}

Major (Bellazzini et al. 2004) or the structure of the outer Galactic young disk, organized as spiral arms (Carraro et al. 2005; Moitinho et al. 2006; Vazquez et al. 2008). Alternatively, these stars can be the blue straggler population of the old, metal-poor population mentioned above. Finally, they can be mostly hot subdwarfs of type $\mathrm{O}$ and $\mathrm{B}$, which are known to be present in the general Galactic field, both in the disk and in the halo (Carraro et al. 2015). Obviously, better scrutiny of these different scenarios can be undertaken only via a dedicated spectroscopic study of these blue stars. This is one of the main objects of this work. We focus here on the line of sight toward the loose open cluster Tombaugh 1 . To set the scene, we show in Figure 1 an optical CMD of a $20 \times 20$ arcmin field, on the $V / V-I$ plane, derived from a novel set of $U B V(\mathrm{RI})_{\mathrm{KC}}$ photometry obtained for the present study. In this CMD, we highlight the features we have been discussing so far with four red boxes. Box A encompasses clump stars in the star cluster Tombaugh 1, which we already studied in Sales Silva et al. (2016), and are not relevant to this study. We remind the reader that Tombaugh 1 turned out to be an intermediate-age ( $\sim$ Gy) open cluster having a metallicity of $[\mathrm{Fe} / \mathrm{H}]=-0.11 \pm 0.02$ and located at $2.6 \mathrm{kpc}$ from the Sun. Boxes B, C, and D are on the other hand central to the present investigation. Box $\mathrm{C}$ encompasses a group of blue stars that are part of the blue plume feature. Box D includes stars belonging to a thick blue MS whose TO is located at $V \sim 19,(V-I) \sim 0.9$. Finally, box B is composed of a clump of scattered stars, possibly red giant stars belonging to the same stellar population of box D. The aim of this work is to characterize these three different groups and establish any possible relation among them. We 


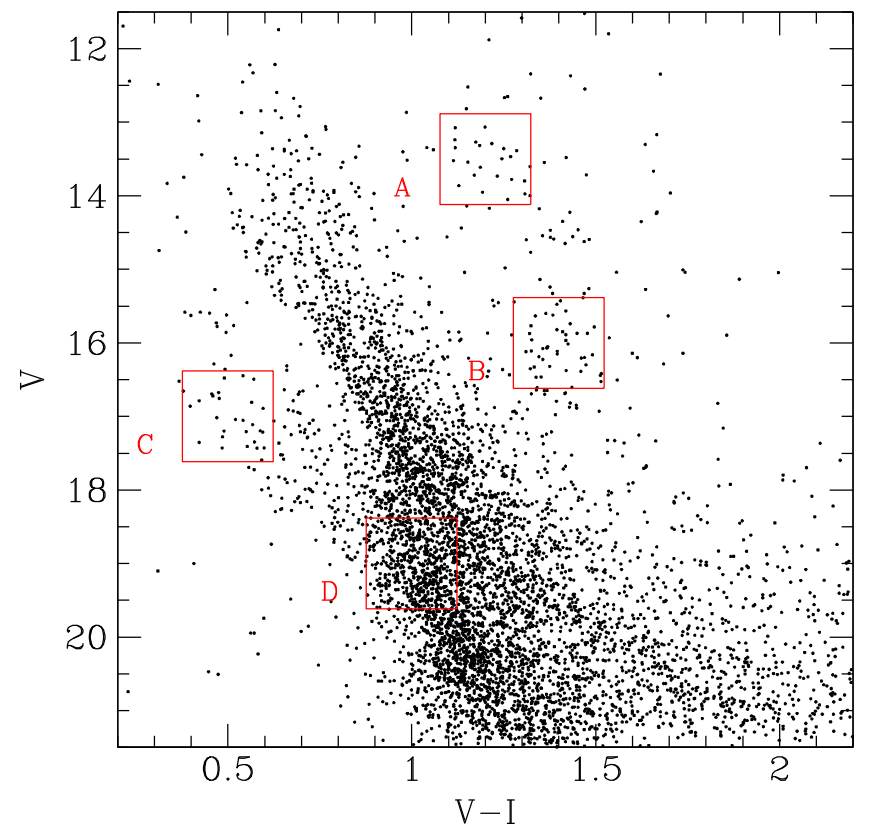

Figure 1. Color-magnitude diagram of the region under study, with the areas highlighted where spectroscopy has been conducted.

selected a sample of stars inside each of these boxes and obtained for them high-resolution spectroscopy, which we are going to present and analyze in tandem with broadband optical photometry.

The paper is organized as follows: In Section 2 we present the observational material, both photometric and spectroscopic. Section 3 is devoted to the analysis of the various photometry diagrams and the derivation of the blue plume stars' individual reddening and distance. A detailed discussion of the spectroscopic data is presented in Section 4, and in Section 5 we focus on the results of the abundance analysis of box B stars. A discussion of our results, and the conclusions of this work are provided in Section 6.

\section{Observations and Data Reduction}

\subsection{Photometry}

The region of interest has been observed with the Y4KCAM camera attached to the $1.0 \mathrm{~m}$ telescope operated by the SMARTS consortium $^{7}$ and located at the Cerro Tololo InterAmerican Observatory (CTIO). This camera is equipped with an STA $4064 \times 4064$ CCD with $15 \mu$ pixels, yielding a scale of 0 !" 289/pixel and a field of view (FOV) of $20^{\prime} \times 20^{\prime}$ at the Cassegrain focus of the CTIO $1.0 \mathrm{~m}$ telescope. The CCD was operated without binning, at a nominal gain of $1.44 \mathrm{e}^{-} / \mathrm{ADU}$, implying a readout noise of $7 \mathrm{e}^{-}$per quadrant (this detector is read by means of four different amplifiers). ${ }^{8}$ As an illustration we show a $V$-band frame in Figure 2.

The observational data were acquired on the night of 2008 January 30, as summarized in Table 1. We observed Landolt's SA $98 U B V(R I)_{\mathrm{KC}}$ standard star area (Landolt 1992; see Table 1) to tie our $U B V R I$ instrumental system to the standard system. The average seeing was 1 !" 0 .

\footnotetext{
7 http://www.astro.yale.edu/smarts/

$8 \mathrm{QE}$ and other detector characteristics can be found at http://www. astronomy.ohio-state.edu/Y4KCam/detector.html.
}

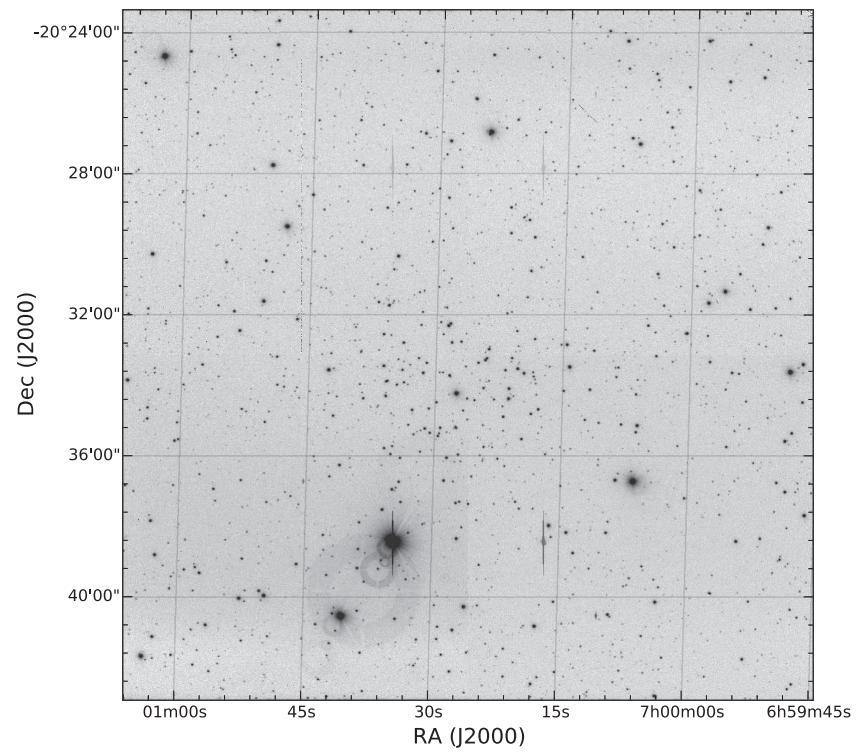

Figure 2. $900 \mathrm{~s} V$-band frame of the area covered by this study.

Our $U B V R I$ instrumental photometric system was defined by the use of a standard broadband Kitt Peak $B V R_{\mathrm{kc}} I_{\mathrm{kc}}$ set in combination with a $\mathrm{U}+\mathrm{CuSO} 4 U$-band filter. ${ }^{9}$ To determine the transformation from our instrumental system to the standard Johnson-Kron-Cousins system, we observed 46 stars in area SA 98 (Landolt 1992) multiple times and with different air masses ranging from $\sim 1.2$ to $\sim 2.3$. Field SA 98 is very advantageous, as it includes a large number of well-observed standard stars, and it is completely covered by the CCD's FOV. Furthermore, the standard's color coverage is very good: $-0.5 \leqslant(U-B) \leqslant 2.2$, $-0.2 \leqslant(B-V) \leqslant 2.2$, and $-0.1 \leqslant(V-I) \leqslant 6.0$.

\subsubsection{Data Reduction}

Basic calibration of the CCD frames was done using the IRAF $^{10}$ package CCDRED. For this purpose, zero-exposure frames and twilight sky flats were acquired every night. Photometry was then performed using the IRAF DAOPHOT and PHOTCAL packages. Instrumental magnitudes were extracted according to the point-spread function (PSF) method (Stetson 1987). A quadratic, spatially variable master PSF (PENNY function) was adopted. The PSF photometry was aperture-corrected, filter by filter. The aperture corrections were determined by performing aperture photometry for a suitable number (typically 20-40) of bright stars selected across the whole field. These corrections were found to vary between 0.105 and $0.315 \mathrm{mag}$, depending on the filter.

\subsubsection{Final Photometry}

Our final photometric catalogs consist of 3275 entries with $U B V(R I)_{\mathrm{KC}}$ measurements down to $V \sim 22$ for Tombaugh 1. Many more entries are available when we include stars without a $U$ magnitude.

After removing both saturated stars and stars having only a few measurements in the catalog of Landolt (1992), we derived

\footnotetext{
9 Transmission curves for these filters can be found at http://www. astronomy.ohio-state.edu/Y4KCam/filters.html.

${ }^{10}$ IRAF is distributed by the National Optical Astronomy Observatory, which is operated by the Association of Universities for Research in Astronomy, Inc., under cooperative agreement with the National Science Foundation.
} 
Table 1

Log of UBVRI Photometric Observations

\begin{tabular}{|c|c|c|c|c|}
\hline Target & Date & Filter & Exposure (s) & Air Mass \\
\hline \multirow[t]{5}{*}{ Tombaugh 1} & 2008 Jan 30 & $\mathrm{U}$ & $10,20,100,200,600,1500$ & $1.28-1.52$ \\
\hline & & $\mathrm{B}$ & $5,10,100,200,1600,1500$ & $1.15-1.20$ \\
\hline & & $\mathrm{V}$ & $5,10,60,120,600,1200$ & $1.01-1.21$ \\
\hline & & $\mathrm{R}$ & $3 \times 5,10,60,120,600,1200$ & $1.02-1.15$ \\
\hline & & I & $5,4 \times 10,100,200,600,1200$ & $1.03-1.24$ \\
\hline \multirow[t]{5}{*}{ SA 98} & 2008 Jan 30 & $\mathrm{U}$ & $2 \times 10,200,2 \times 400$ & $1.15-2.21$ \\
\hline & & $\mathrm{B}$ & $10,100,2 \times 200$ & $1.15-2.36$ \\
\hline & & $\mathrm{V}$ & $10,50,2 \times 100$ & $1.16-2.53$ \\
\hline & & $\mathrm{R}$ & $10,50,2 \times 100$ & $1.17-2.61$ \\
\hline & & I & $10,50,2 \times 100$ & $1.16-2.46$ \\
\hline
\end{tabular}

our photometric solutions for a grand total of 183 measurements in $U$ and $B$ and of 206 measurements in $V, R$, and $I$ :

$$
\begin{aligned}
U= & u+(3.279 \pm 0.010)+(0.47 \pm 0.01) \times X \\
& -(0.030 \pm 0.016) \times(U-B) \\
B= & b+(2.033 \pm 0.012)+(0.29 \pm 0.01) \times X \\
& -(0.110 \pm 0.012) \times(B-V) \\
V= & v+(1.673 \pm 0.007)+(0.16 \pm 0.01) \times X \\
& +(0.022 \pm 0.007) \times(B-V) \\
R= & r+(2.768 \pm 0.007)+(0.10 \pm 0.01) \times X \\
& +(0.053 \pm 0.007) \times(V-R) \\
I= & i+(2.674 \pm 0.011)+(0.08 \pm 0.01) \times X \\
& +(0.048 \pm 0.008) \times(V-I) .
\end{aligned}
$$

The final rms of the fitting was $0.073,0.069,0.035,0.030$, and 0.030 in $U, B, V, R$, and $I$.

Global photometric errors were estimated using the scheme developed by Patat \& Carraro (2001, Appendix A1), which takes into account errors in the PSF fitting procedure (i.e., from ALLSTAR) and calibration errors (corresponding to the zero point, color terms, and extinction errors). In Figure 3 we present the global photometric error trends plotted as a function of $V$ magnitude. A quick inspection indicates that stars brighter than $V \approx 21 \mathrm{mag}$ have errors much smaller than $0.10 \mathrm{mag}$ in both magnitude and color (except for the $(U-B)$ color).

\subsection{Astrometry}

For approximately 300 stars in our photometric catalog, J2000.0 equatorial coordinates are available from the Guide Star Catalog, ${ }^{11}$ version 2 (GSC-2.2, 2001). Using the SkyCat tool at ESO and the IRAF tasks ccxymatch and ccmap, we first established a transformation between our $(X, Y)$ pixel coordinates (from ALLSTAR) and the International Celestial Reference Frame. These transformations have an rms value of typically 0 !' 15 . Finally, using the IRAF task cctran, we computed J2000.0 coordinates for all objects in our catalog.

\subsection{Spectroscopy}

During the nights of 2010 January 5, 6, 9, and 10, we observed 40 stars of the field toward the open cluster Tombaugh 1 (10 stars from boxes A and B, 11 stars from box $\mathrm{C}$, and 9 stars from box D; see Figure 1) on Cerro Manqui at the Las Campanas Observatory using the Inamori-Magellan

\footnotetext{
11 Space Telescope Science Institute, 2001, The Guide Star Catalog Version 2.2.02.
}

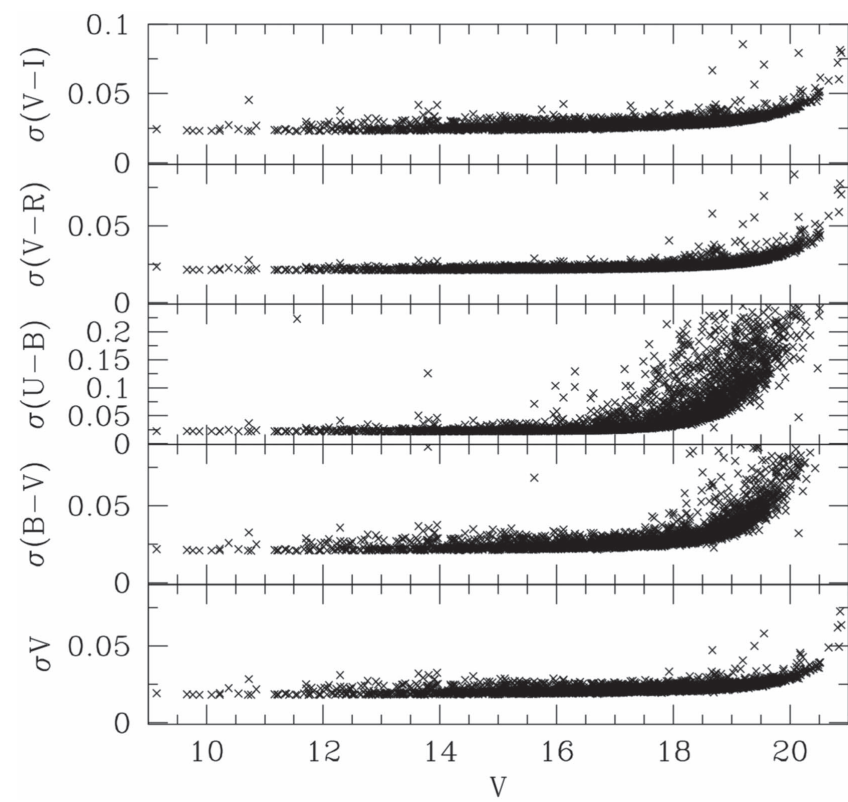

Figure 3. Trends of global photometric errors in color and magnitude as a function of the $V$ magnitude.

Areal Camera \& Spectrograph (IMACS; Dressler et al. 2006), attached to the $6.5 \mathrm{~m}$ Magellan Telescope. The 10 potential cluster stars of box A were studied in Sales Silva et al. (2016). For the stars of boxes A, B, and C, we used the multi-object echelle (MOE) spectroscopic mode, while the spectra of the box D stars were obtained using the multi-object mode with a grating of 600 lines/mm (G600-8.6). The spectra have a resolution of $R \approx 20,000$ and $R \approx 5260$ in the MOE and G600 modes, respectively. In both spectroscopic modes the wavelength ranges of stellar spectra vary according to the position of the star in the observation mask, but usually the wavelength goes from 4200 to $9100 \AA$ for the MOE mode, while for the G600 mode the range is 650 to $6750 \AA$. The IMACS detector is a mosaic of eight CCDs with gaps of $0.93 \mathrm{~mm}$ between them, which cause small gaps in the stellar spectra. The exposure times for the stars of boxes B, C, and D were $9000 \mathrm{~s}, 14,400 \mathrm{~s}$, and $6300 \mathrm{~s}$, divided into three, four, and two exposures, respectively. Table 2 gives some information about the observed stars: identification (ID), equatorial coordinates, $V$ and $(V-I)$ from our photometry, and the spectral signal-tonoise ratio $(\mathrm{S} / \mathrm{N})$ at $6000 \AA$. The identification system for all stars analyzed in this work refers to the identification of stars in our photometry. The nominal $\mathrm{S} / \mathrm{N}$ was evaluated by measuring 
Table 2

Fundamental Information for the Spectroscopically Observed Stars

\begin{tabular}{|c|c|c|c|c|c|}
\hline \multicolumn{6}{|c|}{ Box B } \\
\hline ID & $\begin{array}{l}\text { R.A. }(2000.0) \\
\text { degree }\end{array}$ & $\begin{array}{c}\text { Decl. }(2000.0) \\
\text { degree }\end{array}$ & $\begin{array}{c}V \\
\operatorname{mag}\end{array}$ & $\begin{array}{c}(V-I) \\
\operatorname{mag}\end{array}$ & $\mathrm{S} / \mathrm{N}$ \\
\hline 11029 & 105.0619584 & -20.5959712 & 15.97 & 1.446 & 65 \\
\hline 13540 & 105.0867260 & -20.6172278 & 16.25 & 1.393 & 60 \\
\hline 13964 & 105.0912208 & -20.6830230 & 16.48 & 1.457 & 35 \\
\hline 15490 & 105.1050867 & -20.4189783 & 16.06 & 1.324 & 35 \\
\hline 26606 & 105.1789665 & -20.4134974 & 15.83 & 1.428 & 35 \\
\hline 27955 & 105.1880177 & -20.5694871 & 15.72 & 1.381 & 75 \\
\hline 28064 & 105.1887938 & -20.4728788 & 15.57 & 1.392 & 60 \\
\hline 29403 & 105.1989331 & -20.6387084 & 16.62 & 1.512 & 20 \\
\hline 31364 & 105.2138666 & -20.6505511 & 15.96 & 1.475 & 60 \\
\hline 32782 & 105.2261239 & -20.4283761 & 16.06 & 1.416 & 35 \\
\hline 35658 & 105.2524590 & -20.4933638 & 16.30 & 1.458 & 35 \\
\hline \multicolumn{6}{|c|}{ Box C } \\
\hline 6507 & 105.0154684 & -20.7188840 & 17.31 & 0.549 & 20 \\
\hline 8542 & 105.0351681 & -20.4324223 & 17.21 & 0.566 & 10 \\
\hline 9227 & 105.0426156 & -20.4693299 & 16.82 & 0.458 & 20 \\
\hline 12018 & 105.0719883 & -20.4367526 & 16.46 & 0.491 & 15 \\
\hline 13279 & 105.0840465 & -20.4816043 & 16.88 & 0.676 & 20 \\
\hline 16940 & 105.1177477 & -20.5710991 & 17.24 & 0.653 & 20 \\
\hline 24772 & 105.1683352 & -20.4800548 & 16.77 & 0.474 & 15 \\
\hline 28816 & 105.1943277 & -20.5676972 & 16.58 & 0.489 & 25 \\
\hline 30971 & 105.2109730 & -20.6079883 & 17.06 & 0.695 & 25 \\
\hline 31183 & 105.2125614 & -20.5339462 & 17.30 & 0.696 & 15 \\
\hline 32089 & 105.2197018 & -20.6357812 & 16.41 & 0.659 & 40 \\
\hline \multicolumn{6}{|c|}{ Box D } \\
\hline 7421 & 105.0244349 & -20.5505733 & 19.37 & 1.052 & 15 \\
\hline 9011 & 105.0398536 & -20.6999266 & 18.65 & 0.986 & 20 \\
\hline 9834 & 105.0492119 & -20.4859526 & 19.38 & 1.044 & 10 \\
\hline 11923 & 105.0711463 & -20.4358597 & 19.10 & 0.942 & 10 \\
\hline 19341 & 105.1359171 & -20.4658181 & 19.36 & 1.045 & 10 \\
\hline 22319 & 105.1533307 & -20.5625042 & 18.88 & 1.004 & 20 \\
\hline 23667 & 105.1614867 & -20.4377257 & 18.46 & 0.966 & 15 \\
\hline 36132 & 105.2567499 & -20.4139628 & 18.54 & 0.986 & 10 \\
\hline 31274 & 105.2133773 & -20.5128144 & 19.38 & 1.057 & 10 \\
\hline
\end{tabular}

with IRAF the rms flux fluctuation in selected continuum windows.

Reduction of the spectra was performed in a standard manner under IRAF as described in detail in Sales Silva et al. (2016). The eight CCDs were de-biased and flat-fielded separately with the task ccdproc and combined in a single frame with imcreate and imcopy, and then the spectra were optimum-extracted (Horne 1986) with doecslit (doslit for the G600 mode), skysubtracted with background, and wavelength-calibrated with ecidentify (identify for the G600 spectra). Cosmic rays were removed with the IRAF Laplacian edge detection routine (van Dokkum 2001).

\section{Photometric Diagrams}

We start deriving some insights into the properties of the stellar population in the line of sight to Tombaugh 1 by inspecting a suite of photometric diagrams. Inspection of the CMDs of all the stars in the FOV in Figure 4 reveals three prominent features: (1) a cluster MS with a TO at $B \sim 14.5$ $(V \sim 18.5)$ and a handful of scattered red clump stars at $B \sim 14.3(V \sim 14.2)$ and $(B-R) \sim 1.2$, which we discussed in Sales Silva et al. (2016); (2) a second, thick, well-populated
MS with a TO at $B \sim 19.5$ that looks like the MS of an intermediate-age/old stellar population; and (3) a scattered plume of blue stars in the magnitude range of 16-19 that resembles a young stellar population. The last feature is very similar to the blue plumes found in the directions of other clusters (Carraro et al. 2016 and references therein) in the third Galactic quadrant.

As thoroughly discussed in Carraro et al. (2016), it is quite straightforward to characterize the blue plume, since it would be composed of supposedly young stars, for which a robust handling of the properties is possible with UBV photometry. We discuss the CMD in more detail by plotting stars in different magnitude bins in the classical two-color diagram (TCD) $U-B / B-V$, as shown in Figure 5. A synoptic view of Figures 4 and 5 helps the various CMD components to emerge more clearly, as we have illustrated (see, e.g., Carraro et al. 2010). In the various panels the cyan line is an empirical zero-reddening, zero-age MS (ZAMS) from Turner (1976, 1979). The TCD for $V \leqslant 12$ (lower left panel) only shows a few zero-reddening stars of a different spectral type (from A0 to $\mathrm{F}-\mathrm{G}$ and $\mathrm{M}$ ) located close to the Sun. The middleleft panel is surely more interesting, since on top of the clump of nearby unreddened F-G stars, it shows two groups of reddened stars (spectral types $\mathrm{A}$ and $\mathrm{K}-\mathrm{M}$ ) clearly belonging to the star cluster Tombaugh 1, the first indicating the cluster TO and the second the red giant clump. The upper-left panel samples the Tombaugh 1 TO and MS but also shows a few giant stars, both reddened and unreddened. The next panel (lower right) is by far the most intriguing. One can readily notice an important sequence of early-type, reddened stars totally absent in previous TCDs, which runs from the approximately mid-B spectral type all the way to $\mathrm{K}-\mathrm{M}$. One can also notice (at $(B-V) \sim 0.5,(U-B) \sim 0.0)$ a somewhat detached, truncated, less reddened sequence. The latter is, again, the Tombaugh $1 \mathrm{MS}$, while the more reddened sequence samples the blue plume up to spectral type $\mathrm{AO}$ and later starts to sample a thick blue MS whose TO is at $(B-V) \sim 0.8$. This can be appreciated with the density increase of stars about this color in the TCD. Besides, the giant sequence is also dual and contains zero-reddening nearby giants and more distant, reddened giants possibly associated with the blue thick MS we just described. Finally, the middle-right panel only shows stars in the dominant thick blue MS. The top-right panel is only used to indicate the approximate location of the different spectral type stars.

We focus now on the lower-right panel and attempt to derive the properties of the stars populating the early spectral type branch and corresponding to the blue plume. We obtain a reddening solution for these stars using the TCD in the standard way. This is illustrated in Figure 6.

The reddening law in the third Galactic quadrant has been recently debated in the literature. As discussed by Turner (1976), Moitinho (2001), and Carraro et al. (2016), the reddening law in the third Galactic quadrant seems to deviate from the normal one-namely, it is not described by the standard value of 3.1 for $R_{V}=\frac{A_{V}}{E(B-V)}$. A value of $R_{V}=2.9$ would be more appropriate for this Galactic sector. This is certainly true for the Tombaugh 1 line of sight, as discussed by Turner (1983). Although the level of deviation from the normal law is not significant, we prefer to adopt 2.9 in the following.

The black solid line in Figure 6 is a zero-reddening, zero-age MS, while the two red lines are the same ZAMS, but shifted by 

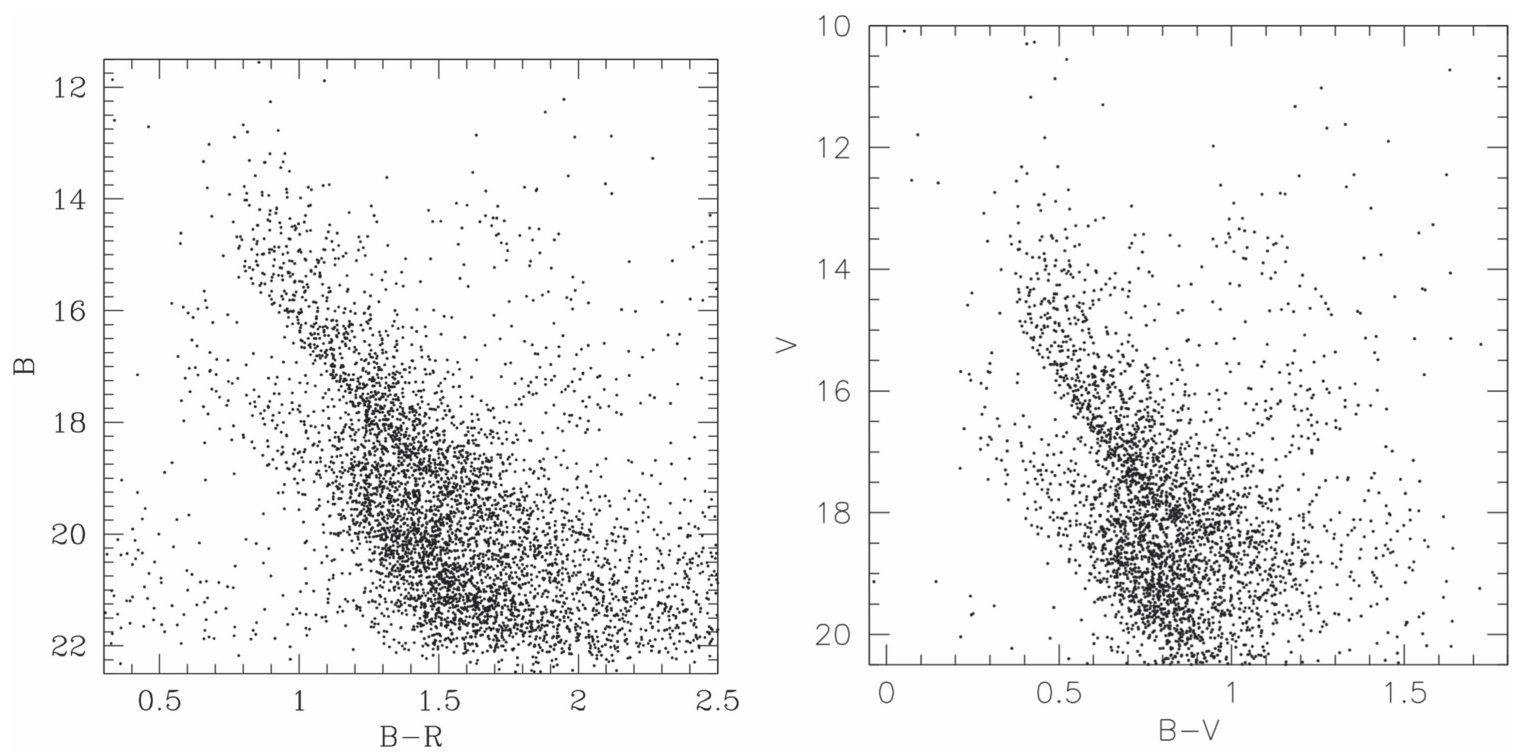

Figure 4. CMDs of the stars in the line of sight to the star cluster Tombaugh 1. On the right panel, the CMD has been constructed using stars having simultaneous measures in $U, B$, and $V$ only, to make the interpretation of Figure 5 easier.

$E(B-V)=0.3$ and 0.7 along the reddening line, which is indicated by the red arrow in the top-right corner of the plot. The two reddened ZAMS bracket the blue plume stars, which therefore exhibit a mean reddening $E(B-V)=0.5 \pm 0.2$, indicating a significant amount of variable reddening. We now analyze the reddening distribution of this population by deriving individual stars' reddening.

To determine reddenings, spectral types, and eventually, distances, we then proceed as follows. First we derive intrinsic colors using the two relationships

$$
E(U-B)=0.76 \times E(B-V)
$$

and

$$
(U-B)_{0}=3.69 \times(B-V)_{0}+0.03 .
$$

The intrinsic color $(B-V)_{0}$ is the positive root of the secondorder equation one derives by combining the above expressions. Intrinsic colors $\left((U-B)_{0}\right.$ and $\left.(B-V)_{0}\right)$ are then directly correlated to spectral type, as compiled, for instance, by Turner (1976, 1979). The solution of the equations above therefore allows us to encounter stars having spectral types earlier than A0.5. For these stars, we know the absolute magnitude $M_{V}$, and from the apparent extinction-corrected magnitude $V_{0}$, we finally infer the photometric distance.

Starting from the general equation for the distance:

$$
(m-M)_{o}=(m-M)_{V}-A_{V}=5 \times \log (\text { Dist })-5,
$$

errors in distances are computed as follows:

$$
\begin{aligned}
& \Delta(\text { Dist })=\ln (10) \times \text { Dist } \times \Delta[\log (\text { Dist })] \\
& \left.\Delta[\log (\text { Dist })]=\frac{1}{5} \times \Delta V+\Delta\left(M_{V}\right)+\Delta\left(A_{V}\right)\right] ; \\
& \Delta\left(M_{V}\right)=0 ; \\
& \Delta\left(A_{V}\right)=2.9 \times \Delta(B-V) ; \\
& \Delta(V) \text { and } \Delta(B-V) \text { directly come from photometry; and } \\
& \text { finally, } \\
& \Delta(\text { Dist })=\ln (10) \times \text { Dist } \times 1 / 5 \times[\Delta V+2.9 \times \\
& \Delta(B-V)] .
\end{aligned}
$$

The reddening distribution is shown on the left panel of Figure 7. It is quite broad and peaks at $E(B-V) \sim 0.5$. A Gaussian fit yields the value $E(B-V)=0.55 \pm 0.20$. On the right panel of the same figure, we show the distribution of the absolute distance moduli for the same stars. Most stars are located in the range $14.0 \leqslant(m-M)_{0} \leqslant 15.5$, which implies a distance range of $6.3 \leqslant d_{\odot} \leqslant 10.0 \mathrm{kpc}$. The errors in distances computed using the formulae described above are $0.5 \mathrm{kpc}$ for the closer stars and up to $1.5 \mathrm{kpc}$ for the most distant stars. We also notice a group of very distant stars, at $(m-M)_{0} \sim 16$, whose distance would be as large as $19 \pm 3.5 \mathrm{kpc}$. We return to this group later.

\section{Spectroscopic Analysis}

\subsection{Radial Velocities}

The radial velocity $(\mathrm{RV})$ of the targets was measured on each single exposure independently. We thus checked for RV variations and shifted them to laboratory wavelengths before co-adding the spectra of each star. The zero-point offset of each spectrum was estimated using the fxcor IRAF task, by crosscorrelating (Tonry \& Davis 1979) the observed telluric band at $6800 \AA$ with that of a FEROS high-resolution solar spectrum we collected in a previous run (Moni Bidin et al. 2012a). The heliocentric correction was estimated through the rvcorrect task and applied to each measurement. The final RV of each star was obtained from the weighted mean of the single epochs. Our results are given in Table 3.

For the box B stars, the line-to-line differences between the observed and laboratory wavelengths of the unblended Fe lines were used to determine the target RV. The final error was assumed as the largest difference between the three heliocentric RV values multiplied by 0.59 (small-sample statistics; see Keeping 1962). The RV of the box C stars was estimated by cross-correlating the $\mathrm{H}_{\alpha}$ line with the synthetic template of a 10,000 K MS star taken from the Munari et al. (2005) library. Previous works have shown that the results of cross-correlation are not affected by the exact choice of template, and a marginal mismatch between object and template spectral types only 

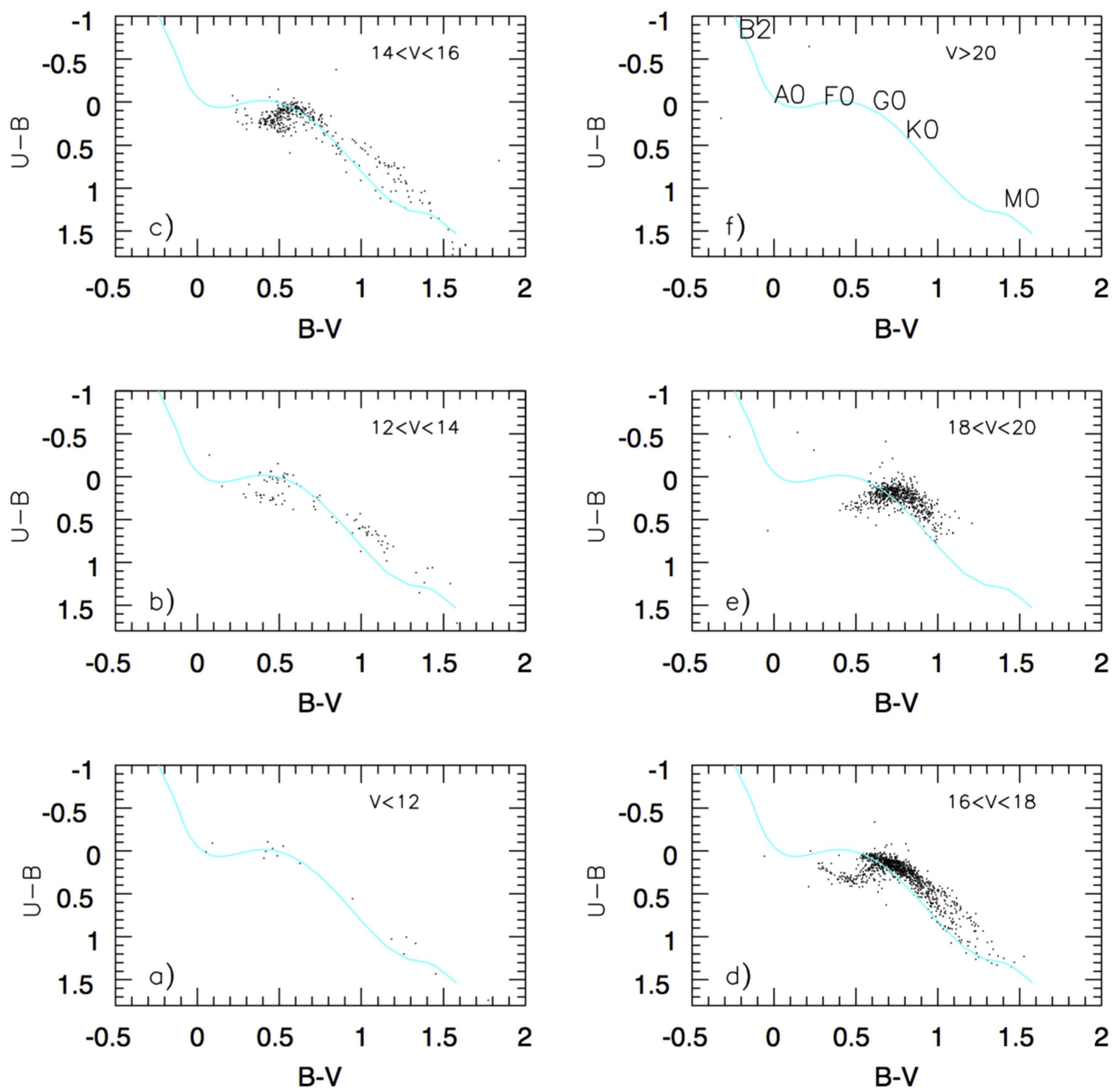

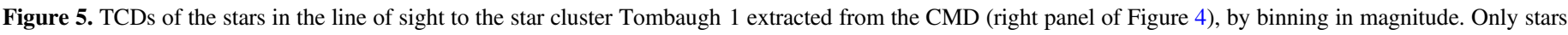
having $U, B$, and $V$ measures simultaneously are plotted.

enhances the formal uncertainties (Morse et al. 1991; Moni Bidin et al. 2011). When other strong features were visible in the spectral range, such as the $\mathrm{H}_{\beta}$ line, we verified that they returned identical results within the errors. However, as the availability of these features varied among the targets, for the sake of consistency we used only the $\mathrm{H}_{\alpha}$ line to derive the final results. The same procedure was adopted for the box D stars, except that the aforementioned FEROS solar spectrum was used as a template.

\subsection{Box B: Stellar Parameters and Chemical Abundances}

The line list used to determine the chemical abundance of $\mathrm{Na}$, $\mathrm{Al}, \mathrm{Mg}, \mathrm{Si}, \mathrm{Ca}, \mathrm{Ti}, \mathrm{Cr}, \mathrm{Fe}$, and $\mathrm{Ni}$ (see Table 4 for the measures and Table 5 for the associates uncertainties) is the same one we used recently in Sales Silva et al. (2016). In Tables 6 and 7 we show our line list with the excitation potential $(\chi)$ and oscillator strength (gf) for all absorption lines analyzed in this work. The values of the oscillator strength adopted for the Fe I and Fe II lines were taken from Lambert et al. (1996) and Castro et al. (1997).
The references of the atomic parameters for the other absorption lines are shown in Table 7.

The chemical abundance of $\mathrm{Na}, \mathrm{Al}, \mathrm{Mg}, \mathrm{Si}, \mathrm{Ca}, \mathrm{Ti}, \mathrm{Cr}$, and $\mathrm{Ni}$ for the red clump stars (box B) was obtained through the equivalent widths (EWs) of the absorption lines corresponding to each element. The EWs were measured using the task splot in IRAF by fitting the observed absorption line with a Gaussian profile. Absorption lines with EWs greater than $160 \mathrm{m \AA}$ are saturated and were rejected in our analysis due to the impossibility of fitting a Gaussian profile to these lines (Pereira et al. 2011). The EWs used to derive the chemical abundance are shown in Tables 6 and 7.

The local thermodynamic equilibrium (LTE) model atmospheres of Kurucz (1993) and the spectral analysis code MOOG (Sneden 1973) were used to determine the chemical abundances and atmospheric parameters for the stars of box B. The effective temperature, surface gravity, and microturbulence velocity were derived using measurements of EWs for a set of $\mathrm{Fe} \mathrm{I}$ and Fe II lines, shown in Table 6. The unique solutions for 


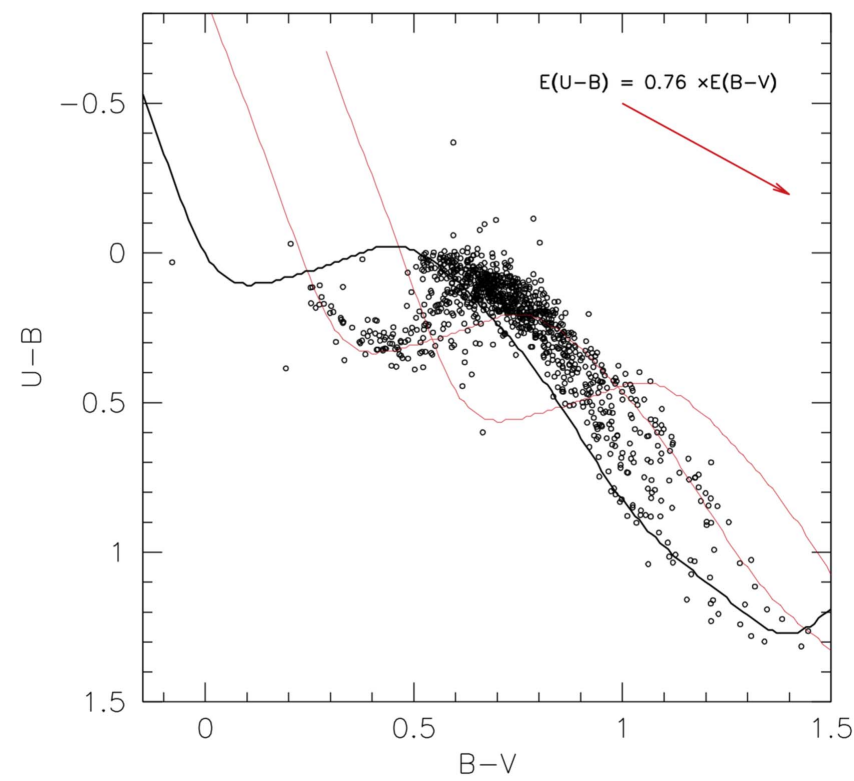

Figure 6. TCDs of the stars in the line of sight to the star cluster Tombaugh 1 and in the magnitude range $16 \leqslant V \leqslant 18$. The solid line is a zero-reddening, zero-age main sequence, while the two red lines encompassing the early-type stars are the same ZAMS, but displaced by 0.4 and 0.6 mag along the reddening vector.

the effective temperature, surface gravity, and microturbulence velocity were obtained simultaneously under approximations of excitation and ionization equilibrium and independence between the Fe abundance and reduced EW. The effective temperature was set through the excitation equilibrium determined by a zero slope of the trend between the iron abundance derived from Fe I lines and the excitation potential of the measured lines. The microturbulence velocity was adjusted until both the strong and weak Fe I lines gave the same abundance. The ionization equilibrium was used to derive the surface gravity and was defined by the equality of the abundances of Fe I and Fe II.

Uncertainties in the effective temperature and microturbulence velocity were inferred from the uncertainties in the slopes of the Fe I abundances versus the potential excitation and the abundance of $\mathrm{Fe} I$ versus the reduced $\mathrm{EW}$, respectively. On the other hand, the uncertainty in surface gravity was obtained by varying this parameter iteratively around the first-guess value until surface gravity was obtained that changes the abundance of Fe II by exactly one standard deviation of the $[\mathrm{Fe} \mathrm{I} / \mathrm{H}]$ mean value. In Table 3 we show the atmospheric parameters and their respective uncertainties for the red clump stars.

The atmospheric parameters for stars \#15490, \#29403, and \#35658 were not determined because the spectra of these stars have low $\mathrm{S} / \mathrm{N}$. Despite the low $\mathrm{S} / \mathrm{N}$ also shown by the spectra of stars \#13964 and \#32782, we could derive their atmospheric parameters, but with large uncertainty (see Table 3). In the analysis of star \#26606, we faced a problem in obtaining the microturbulence velocity due to the low number of absorption lines with small EW, which again caused considerable uncertainty in the atmospheric parameters.

In Table 4 we show the abundance ratios ([X/Fe]) for $\mathrm{Na}$, $\mathrm{Al}, \mathrm{Mg}, \mathrm{Ca}, \mathrm{Si}, \mathrm{Ti}, \mathrm{Cr}$, and $\mathrm{Ni}$ for the red clump stars. Our chemical abundances were normalized to the solar abundances obtained through a high-resolution FEROS solar spectrum (Moni Bidin et al. 2012a). In Table 8 we list our solar abundances and those of Grevesse \& Sauval (1998) and Asplund et al. (2009). The total uncertainties of the abundances for the red clump stars are shown in Table 5. The uncertainties of the chemical abundances associated with the errors in the effective temperature, microturbulence velocity, and surface gravity were calculated independently and then quadratically combined to provide the global abundance uncertainty.

\subsection{Boxes $C$ and D: Stellar Parameters}

The temperature, gravity, and rotational velocity of box $\mathrm{C}$ stars were measured by fitting the $\mathrm{H}_{\alpha}$ and $\mathrm{H}_{\beta}$ Balmer lines and the $4922 \AA$ He I feature with synthetic spectra, as was done in Majaess et al. (2013) for similar MS stars. With this aim, we employed the fitprof 21 code, developed by Bergeron et al. (1992) and Saffer et al. (1994) and subsequently modified by Napiwotzki et al. (1999). The routine was fed with a grid of solar-metallicity LTE model spectra $\left(T_{\text {eff }}=8000-30,000 \mathrm{~K}\right.$, $\log g=3.5-5.0$ dex) generated from ATLAS9 (Kurucz 1993) model atmospheres through the Lemkes version ${ }^{12}$ of the LINFOR program (developed originally by Holweger, Steffen, and Steenbock at Kiel University). In fact, deviations from LTE have negligible effects on the Balmer and He lines at the temperatures of program stars (Nieva \& Przybilla 2007). The routine determines the best-fit parameters through $\chi^{2}$ minimization statistics. Extensive details about the synthetic spectral fitting procedure can be found in Moni Bidin et al. (2012b).

While the method ideally works when the whole Balmer series can be simultaneously fit, a minimum of two features are required to avoid degeneracy between the temperature and gravity. In our case, the profile of the only $\mathrm{He}$ line could constrain the rotational velocity and, to a limited extent, the temperature, but the two Balmer lines were needed for a reliable determination of $T_{\text {eff }}$ and $\log g$. This was not possible for the three targets, where the $\mathrm{H}_{\beta}$ line did not fall in the spectral range. We could still obtain a good fit of the single $\mathrm{H}_{\alpha}$ line for stars \#6507 and \#8542, although the larger errors reflect the high uncertainty of the measurements. For star $\# 12018$, on the other hand, we had to assume $\log g=4.2$, as is typical of an MS star, and adopt only the temperature as the fit parameter. The strength of the Balmer lines has a maximum at $\approx 10,000 \mathrm{~K}$, and it declines for both hotter and cooler stars. The temperatures obtained by one Balmer line only could thus present two acceptable solutions, symmetric with respect to the A0 spectral type. We therefore analyzed the results of the three aforementioned stars in more detail. We indeed found a secondary solution for star \#8542, with a local minimum of the $\chi^{2}$ statistics, on the other side of the Balmer minimum at $10,100 \mathrm{~K}$. However, this minimum of $\chi^{2}$ is lower than the main solution at $8100 \mathrm{~K}$, which is therefore more likely and should be preferred. We did not find such a secondary minimum for star \#12018, and the fitting routine converged to the same solution at $8700 \mathrm{~K}$ even if it had been forced to start from a hot first guess at $T_{\text {eff }}>12,000 \mathrm{~K}$. On the other hand, a cooler solution for star \#6507 is not acceptable, because $T_{\text {eff }}<9000$ $\mathrm{K}$ would return $E(V-I)<0.37$ and a distance $d \approx 10 \mathrm{kpc}$, clearly offset from the reddening-distance relation depicted by

\footnotetext{
${ }^{12}$ http://www.sternwarte.uni-erlangen.de/ãi26/linfit/linfor.html
} 

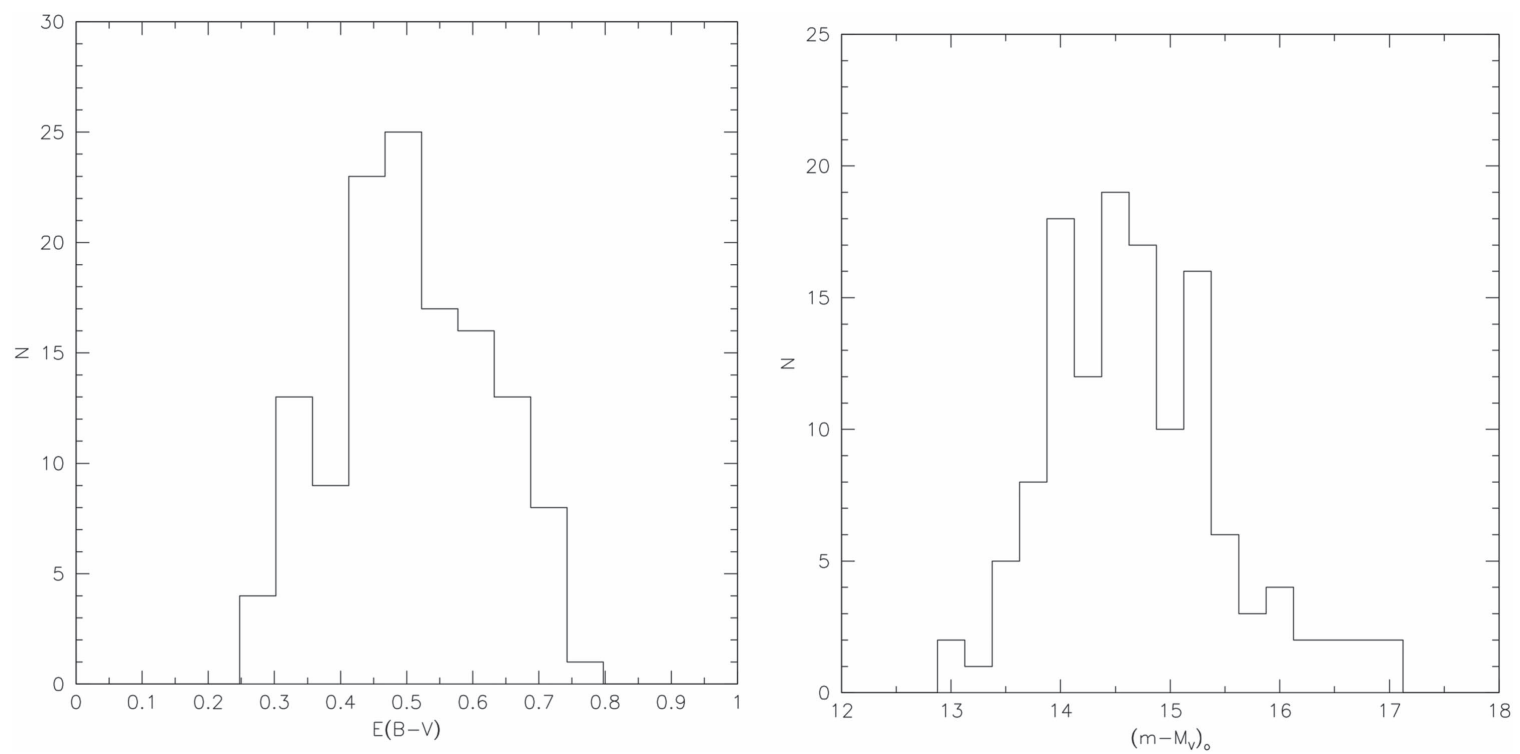

Figure 7. Distribution of individual star reddenings (left panel) and reddening-corrected distance moduli (right panel).

Table 3

Atmospheric Parameters from Spectroscopy of Stars of Box B

\begin{tabular}{|c|c|c|c|c|c|c|c|c|c|}
\hline ID & $\begin{array}{l}T_{\text {eff }} \\
(\mathrm{K})\end{array}$ & $\begin{array}{l}\log g \\
(\mathrm{dex})\end{array}$ & $\begin{array}{c}\xi \\
\left(\mathrm{km} \mathrm{s}^{-1}\right)\end{array}$ & {$[\mathrm{Fe} \mathrm{I} / \mathrm{H}] \pm \sigma(\#)$} & {$[\mathrm{Fe} \mathrm{II} / \mathrm{H}] \pm \sigma(\#)$} & $\underset{\left(\mathrm{km} \mathrm{s}^{-1}\right)}{\langle\mathrm{RV}\rangle}$ & $E(V-I)$ & $(V-M v)_{0}$ & $\begin{array}{c}d \\
(\mathrm{pc})\end{array}$ \\
\hline 11029 & $5250 \pm 200$ & $3.3 \pm 0.2$ & $2.2 \pm 0.4$ & $-0.03 \pm 0.13(43)$ & $-0.03 \pm 0.12(8)$ & $76.2 \pm 1.4$ & $0.53 \pm 0.19$ & $12.41 \pm 0.61$ & $3000 \pm 900$ \\
\hline 13540 & $5300 \pm 200$ & $3.1 \pm 0.2$ & $1.8 \pm 0.3$ & $0.03 \pm 0.14(27)$ & $0.05 \pm 0.07(3)$ & $74.6 \pm 1.8$ & $0.56 \pm 0.11$ & $13.62 \pm 0.80$ & $5300 \pm 2000$ \\
\hline $13964^{\mathrm{a}}$ & $4700 \pm 300$ & $2.0 \pm 0.4$ & $2.5 \pm 0.6$ & $-0.68 \pm 0.18(23)$ & $-0.70 \pm 0.18(3)$ & $73.9 \pm 2.1$ & $0.39 \pm 0.19$ & $16.55 \pm 1.17$ & $20500 \pm 11600$ \\
\hline $26606^{\mathrm{b}}$ & $4600 \pm 450$ & $2.7 \pm 0.5$ & $2.5 \pm 1.0$ & $-0.46 \pm 0.34(19)$ & $-0.45 \pm 0.26(3)$ & $28.1 \pm 3.6$ & $0.30 \pm 0.25$ & $13.04 \pm 1.09$ & $4000 \pm 2100$ \\
\hline 27955 & $5250 \pm 250$ & $3.8 \pm 0.2$ & $2.2 \pm 0.4$ & $-0.24 \pm 0.15(35)$ & $-0.24 \pm 0.08(6)$ & $116.5 \pm 1.3$ & $0.53 \pm 0.10$ & $10.46 \pm 0.57$ & $1200 \pm 300$ \\
\hline 28064 & $4700 \pm 150$ & $2.1 \pm 0.3$ & $2.3 \pm 0.4$ & $-0.58 \pm 0.14(33)$ & $-0.57 \pm 0.15(5)$ & $68.0 \pm 3.2$ & $0.37 \pm 0.15$ & $15.45 \pm 0.89$ & $12300 \pm 5200$ \\
\hline 31364 & $5050 \pm 150$ & $2.0 \pm 0.2$ & $1.5 \pm 0.3$ & $-0.24 \pm 0.13(31)$ & $-0.23 \pm 0.08(3)$ & $65.6 \pm 0.3$ & $0.44 \pm 0.09$ & $16.81 \pm 0.90$ & $23000 \pm 980$ \\
\hline 32782 & $5000 \pm 250$ & $3.1 \pm 0.3$ & $3.0 \pm 0.6$ & $-0.39 \pm 0.13(16)$ & $-0.41(1)$ & $0.9 \pm 3.0$ & $0.49 \pm 0.14$ & $12.79 \pm 0.48$ & $3600 \pm 800$ \\
\hline
\end{tabular}

Notes. For $[\mathrm{Fe} \mathrm{I} / \mathrm{H}]$ and $[\mathrm{Fe} \mathrm{II} / \mathrm{H}]$, we also show the standard deviation and the number of lines (\#) employed.

${ }^{a}$ Large uncertainty in the atmospheric parameters and metallicity of this star due to low $\mathrm{S} / \mathrm{N}$.

${ }^{\mathrm{b}}$ Problem in obtaining the microturbulent velocity $(\xi)$ due to the low number of absorption lines with a small equivalent width, causing considerable uncertainty in the metallicity.

the other stars in boxes C and D (see below in Figure 10). In addition, this object is of little interest because it is most likely not a genuine MS star (see below). In conclusion, although we fit one Balmer line only, the solutions we found for these three stars are either the only ones acceptable or the most likely ones. To check the effects of the use of solar-metallicity models, we repeated the measurements with models with $[\mathrm{Fe} / \mathrm{H}]=-0.5$, but we found that the results changed by less than $0.5 \sigma$ in all cases. The resulting surface gravities indicate that all the targets are MS stars, with the exception of star \#6507. The measurements for this star are affected by large errors, so that it could still be considered an MS object within the error bars, but the high $\log g$ value suggests that this could be a foreground subdwarf B-type (sdB) star.

The temperature of box D targets was determined by fitting the profile of temperature-sensitive lines with synthetic spectra drawn from the library of Coelho et al. (2005). We adopted the same routines from Moni Bidin et al. (2010), where detailed information about the measurement procedure can be found.
The main feature for our measurements was the $\mathrm{H}_{\alpha}$ Balmer line, which is a good indicator of temperature in the range $T_{\text {eff }}=5000-6500 \mathrm{~K}$, insensitive to metallicity and surface gravity (Fuhrmann et al. 1994). Its wings were fitted with solarmetallicity templates in steps of $250 \mathrm{~K}$, and the $\chi^{2}$ was minimized to find the best estimate of the target temperature. We verified that varying the metallicity of the synthetic templates had only negligible effects on the results.

One gravity-sensitive feature was observable in the spectra of some targets, either the $\mathrm{Mg} \mathrm{Ib}$ triplet (Kuijken \& Gilmore 1989) or the Na I doublet at 5890-5893 A. However, the low resolution of the data precluded an estimate of $\log g$, because only large mismatches ( $>1$ dex) between the template and object line wings could be appreciated. Hence, the targets were assumed MS stars throughout the process, with a fixed surface gravity of $\log g=4.1 \mathrm{dex}$, as strongly suggested by their position in the CMD. We nevertheless confirmed this hypothesis by checking that the profiles of available lines were compatible with it. On the other hand, this assumption was also 
Table 4

Abundance Ratios ([X/Fe]) for the Elements, from Na to Cr, for the Stars from Box B

\begin{tabular}{|c|c|c|c|c|c|c|c|c|}
\hline \multicolumn{9}{|c|}{ Box B } \\
\hline ID & {$[\mathrm{Na} / \mathrm{Fe}] \mathrm{NLTE}$} & {$[\mathrm{Mg} / \mathrm{Fe}]$} & {$[\mathrm{Al} / \mathrm{Fe}]$} & {$[\mathrm{Si} / \mathrm{Fe}]$} & {$[\mathrm{Ca} / \mathrm{Fe}]$} & {$[\mathrm{Ti} / \mathrm{Fe}]$} & {$[\mathrm{Cr} / \mathrm{Fe}]$} & {$[\mathrm{Ni} / \mathrm{Fe}]$} \\
\hline 11029 & $+0.19(1)$ & $+0.18(1)$ & $\ldots$ & $+0.13 \pm 0.05(2)$ & $-0.20 \pm 0.12(4)$ & $+0.29 \pm 0.10(5)$ & $-0.09 \pm 0.02(3)$ & $-0.08 \pm 0.09(10)$ \\
\hline 13540 & $+0.27 \pm 0.01(2)$ & $\ldots$ & $+0.14 \pm 0.04(2)$ & $+0.12 \pm 0.16(3)$ & $+0.18 \pm 0.11(3)$ & $+0.29 \pm 0.04(3)$ & $-0.21 \pm 0.10(3)$ & $+0.08 \pm 0.13(10)$ \\
\hline 13964 & $+0.40(1)$ & $+0.66(1)$ & $+0.37 \pm 0.01(2)$ & $\ldots$ & $+0.47 \pm 0.07(4)$ & $+0.21 \pm 0.14(6)$ & $+0.35(1)$ & $+0.24 \pm 0.14(4)$ \\
\hline 26606 & $\ldots$ & $+0.41 \pm 0.06(2)$ & $+0.47(1)$ & $+0.54(1)$ & $\ldots$ & $+0.23 \pm 0.03(2)$ & $+0.12(1)$ & $+0.01(1)$ \\
\hline 27955 & $\ldots$ & $+0.00 \pm 0.13(2)$ & $\ldots$ & $+0.11 \pm 0.10(2)$ & $-0.17 \pm 0.17(4)$ & $+0.37 \pm 0.08(5)$ & $-0.11 \pm 0.03(3)$ & $+0.17 \pm 0.16(11)$ \\
\hline 28064 & $+0.38(1)$ & $+0.24 \pm 0.08(2)$ & $+0.10 \pm 0.11(4)$ & $+0.12(1)$ & $-0.30 \pm 0.03(2)$ & $+0.22 \pm 0.13(4)$ & $+0.10 \pm 0.13(2)$ & $-0.22 \pm 0.07(7)$ \\
\hline 31364 & $+0.37(1)$ & $-0.21 \pm 0.10(2)$ & & $+0.07 \pm 0.17(3)$ & $+0.03 \pm 0.11(3)$ & $+0.31 \pm 0.15(3)$ & $+0.07 \pm 0.02(2)$ & $-0.11 \pm 0.16(10)$ \\
\hline 32782 & $+0.42(1)$ & $+0.67(1)$ & $+0.59(1)$ & $\ldots$ & $\ldots$ & $+0.54 \pm 0.14(3)$ & $+0.10(1)$ & $-0.02 \pm 0.15(5)$ \\
\hline
\end{tabular}

Note. For all abundances ratios, we also show the standard deviation and the number of lines employed. [Na/Fe] accounts for the NLTE effects calculated as in Gratton et al. (1999)—see the text. 
Table 5

Abundance Uncertainties for Stars from Box B

\begin{tabular}{|c|c|c|c|c|c|c|c|c|}
\hline \multirow[b]{2}{*}{ Element } & \multicolumn{4}{|c|}{11029} & \multicolumn{4}{|c|}{13540} \\
\hline & $\begin{array}{c}\Delta T_{\text {eff }} \\
+200 \mathrm{~K}\end{array}$ & $\begin{array}{l}\Delta \log g \\
+0.2\end{array}$ & $\begin{array}{c}\Delta \xi \\
+0.4 \mathrm{~km} \mathrm{~s}^{-1}\end{array}$ & $\left(\sum \sigma^{2}\right)^{1 / 2}$ & $\begin{aligned} & \Delta T_{\text {eff }} \\
+ & 200 \mathrm{~K}\end{aligned}$ & $\begin{array}{l}\Delta \log g \\
+0.2\end{array}$ & $\begin{array}{c}\Delta \xi \\
+0.3 \mathrm{~km} \mathrm{~s}^{-1}\end{array}$ & $\left(\sum \sigma^{2}\right)^{1 / 2}$ \\
\hline $\mathrm{Fe} I$ & +0.13 & 0.00 & -0.11 & 0.17 & +0.15 & -0.01 & -0.13 & 0.20 \\
\hline Fe II & -0.17 & +0.08 & -0.11 & 0.22 & -0.15 & +0.10 & -0.12 & 0.22 \\
\hline $\mathrm{Na} I$ & +0.13 & 0.00 & -0.06 & 0.14 & +0.14 & -0.01 & -0.07 & 0.16 \\
\hline Mg I & +0.13 & -0.02 & -0.16 & 0.21 & $\ldots$ & $\ldots$ & $\ldots$ & $\ldots$ \\
\hline $\mathrm{Al} \mathrm{I}$ & $\ldots$ & $\ldots$ & $\ldots$ & $\ldots$ & +0.08 & -0.01 & -0.04 & 0.09 \\
\hline Si I & -0.04 & +0.02 & -0.04 & 0.06 & +0.01 & +0.02 & -0.06 & 0.06 \\
\hline $\mathrm{Ca} \mathrm{I}$ & +0.18 & -0.02 & -0.15 & 0.24 & +0.18 & -0.02 & -0.16 & 0.24 \\
\hline Ti I & +0.25 & 0.00 & -0.11 & 0.27 & +0.23 & -0.01 & -0.11 & 0.26 \\
\hline Cr I & +0.25 & -0.01 & -0.20 & 0.32 & +0.24 & -0.01 & -0.12 & 0.27 \\
\hline \multirow[t]{2}{*}{ Ni I } & +0.09 & +0.02 & -0.12 & 0.15 & +0.12 & +0.01 & -0.13 & 0.18 \\
\hline & \multicolumn{4}{|c|}{13964} & \multicolumn{4}{|c|}{26606} \\
\hline Element & $\begin{array}{c}\Delta T_{\text {eff }} \\
+300 \mathrm{~K}\end{array}$ & $\begin{array}{l}\Delta \log g \\
+0.4\end{array}$ & $\begin{array}{c}\Delta \xi \\
+0.6 \mathrm{~km} \mathrm{~s}^{-1}\end{array}$ & $\left(\sum \sigma^{2}\right)^{1 / 2}$ & $\begin{array}{c}\Delta T_{\text {eff }} \\
+450 \mathrm{~K}\end{array}$ & $\begin{array}{c}\Delta \log g \\
+0.5\end{array}$ & $\begin{array}{c}\Delta \xi \\
+1.0 \mathrm{~km} \mathrm{~s}^{-1}\end{array}$ & $\left(\sum \sigma^{2}\right)^{1 / 2}$ \\
\hline $\mathrm{Fe} I$ & +0.18 & -0.03 & -0.16 & 0.24 & +0.34 & -0.06 & -0.35 & 0.49 \\
\hline $\mathrm{Fe}$ II & -0.35 & +0.22 & -0.10 & 0.43 & -0.36 & +0.23 & -0.17 & 0.46 \\
\hline $\mathrm{Na} I$ & +0.26 & -0.01 & -0.09 & 0.28 & $\ldots$ & $\ldots$ & $\ldots$ & $\ldots$ \\
\hline Mg I & +0.07 & -0.02 & -0.12 & 0.14 & +0.12 & 0.00 & -0.16 & 0.20 \\
\hline $\mathrm{Al} \mathrm{I}$ & +0.15 & -0.01 & -0.04 & 0.16 & +0.32 & -0.01 & -0.16 & 0.36 \\
\hline Si I & $\ldots$ & $\ldots$ & $\ldots$ & $\ldots$ & +0.24 & +0.09 & -0.19 & 0.32 \\
\hline $\mathrm{Ca} \mathrm{I}$ & +0.35 & -0.01 & -0.25 & 0.43 & $\ldots$ & $\ldots$ & $\ldots$ & $\cdots$ \\
\hline Ti I & +0.50 & -0.01 & -0.13 & 0.52 & +0.68 & 0.00 & -0.59 & 0.90 \\
\hline Cr I & +0.28 & -0.01 & -0.08 & 0.29 & +0.36 & 0.00 & -0.10 & 0.37 \\
\hline \multirow[t]{2}{*}{$\mathrm{Ni} \mathrm{I}$} & +0.05 & +0.06 & -0.09 & 0.12 & +0.13 & +0.10 & -0.33 & 0.37 \\
\hline & \multicolumn{4}{|c|}{27955} & \multicolumn{4}{|c|}{28064} \\
\hline Element & $\begin{array}{c}\Delta T_{\text {eff }} \\
+250 \mathrm{~K}\end{array}$ & $\begin{array}{c}\Delta \log g \\
+0.2\end{array}$ & $\begin{array}{c}\Delta \xi \\
+0.4 \mathrm{~km} \mathrm{~s}^{-1}\end{array}$ & $\left(\sum \sigma^{2}\right)^{1 / 2}$ & $\begin{array}{c}\Delta T_{\text {eff }} \\
+150 \mathrm{~K}\end{array}$ & $\begin{array}{c}\Delta \log g \\
+0.3\end{array}$ & $\begin{array}{c}\Delta \xi \\
+0.4 \mathrm{~km} \mathrm{~s}^{-1}\end{array}$ & $\left(\sum \sigma^{2}\right)^{1 / 2}$ \\
\hline $\mathrm{Fe} I$ & +0.14 & 0.00 & -0.11 & 0.18 & +0.14 & +0.03 & -0.13 & 0.19 \\
\hline Fe II & -0.18 & +0.10 & -0.08 & 0.22 & -0.11 & +0.16 & -0.07 & 0.21 \\
\hline $\mathrm{Na} I$ & $\ldots$ & $\ldots$ & $\ldots$ & $\ldots$ & +0.12 & -0.01 & -0.05 & 0.13 \\
\hline $\mathrm{Mg} \mathrm{I}$ & +0.10 & -0.02 & -0.07 & 0.12 & +0.05 & 0.00 & -0.07 & 0.09 \\
\hline $\mathrm{Al} \mathrm{I}$ & $\ldots$ & $\ldots$ & $\ldots$ & $\ldots$ & +0.08 & -0.01 & -0.04 & 0.09 \\
\hline Si I & +0.06 & +0.03 & -0.04 & 0.08 & -0.05 & +0.07 & -0.09 & 0.12 \\
\hline $\mathrm{Ca} \mathrm{I}$ & +0.22 & -0.02 & -0.12 & 0.25 & +0.18 & -0.01 & -0.21 & 0.28 \\
\hline Ti I & +0.30 & -0.01 & -0.15 & 0.34 & +0.23 & -0.01 & -0.09 & 0.25 \\
\hline Cr I & +0.24 & -0.01 & -0.10 & 0.26 & +0.15 & -0.01 & -0.10 & 0.18 \\
\hline \multirow[t]{2}{*}{ Ni I } & +0.10 & +0.02 & -0.12 & 0.16 & +0.10 & +0.05 & -0.18 & 0.21 \\
\hline & \multicolumn{4}{|c|}{31364} & \multicolumn{4}{|c|}{32782} \\
\hline Element & $\begin{array}{c}\Delta T_{\text {eff }} \\
+150 \mathrm{~K}\end{array}$ & $\begin{array}{c}\Delta \log g \\
+0.2\end{array}$ & $\begin{array}{c}\Delta \xi \\
+0.3 \mathrm{~km} \mathrm{~s}^{-1}\end{array}$ & $\left(\sum \sigma^{2}\right)^{1 / 2}$ & $\begin{array}{c}\Delta T_{\text {eff }} \\
+250 \mathrm{~K}\end{array}$ & $\begin{array}{c}\Delta \log g \\
+0.3\end{array}$ & $\begin{array}{c}\Delta \xi \\
+0.6 \mathrm{~km} \mathrm{~s}^{-1}\end{array}$ & $\left(\sum \sigma^{2}\right)^{1 / 2}$ \\
\hline $\mathrm{Fe} I$ & +0.14 & 0.00 & -0.12 & 0.18 & +0.15 & 0.00 & -0.14 & 0.21 \\
\hline $\mathrm{Fe}$ II & -0.12 & +0.13 & -0.13 & 0.22 & -0.21 & +0.13 & -0.09 & 0.26 \\
\hline $\mathrm{Na} I$ & +0.11 & -0.01 & -0.03 & 0.11 & +0.19 & -0.01 & -0.08 & 0.21 \\
\hline Mg I & +0.05 & 0.00 & -0.05 & 0.07 & +0.04 & -0.06 & -0.10 & 0.12 \\
\hline $\mathrm{Al} \mathrm{I}$ & $\ldots$ & $\ldots$ & $\ldots$ & $\ldots$ & +0.11 & -0.01 & -0.06 & 0.13 \\
\hline Si I & +0.01 & +0.03 & -0.04 & 0.05 & $\ldots$ & $\ldots$ & $\ldots$ & $\ldots$ \\
\hline $\mathrm{Ca} \mathrm{I}$ & +0.16 & -0.01 & -0.17 & 0.23 & $\ldots$ & $\ldots$ & $\ldots$ & $\ldots$ \\
\hline Ti I & +0.22 & -0.01 & -0.09 & 0.24 & +0.34 & 0.00 & -0.11 & 0.36 \\
\hline $\mathrm{Cr} \mathrm{I}$ & +0.25 & -0.02 & -0.17 & 0.30 & +0.36 & 0.00 & -0.10 & 0.37 \\
\hline $\mathrm{Ni} I$ & +0.14 & +0.02 & -0.12 & 0.19 & +0.11 & +0.04 & -0.15 & 0.19 \\
\hline
\end{tabular}

Note. Each column gives the variation of the abundance caused by the variation in $T_{\text {eff }}, \log g$, and $\xi$. The last column for each star gives the compounded rms uncertainty of the second to fourth columns.

confirmed by later distance estimates because had one of these stars been either a faint subdwarf or a bright giant, its distance would have been extremely large or small, respectively, which is not the case. The $\mathrm{Mg} \mathrm{Ib}$ triplet and the $\mathrm{Na}$ I doublet were instead used to derive independent estimates of $T_{\text {eff }}$, with a procedure identical to that used for $\mathrm{H}_{\alpha}$. The final results and 
Table 6

Observed Fe I and Fe II Lines

\begin{tabular}{|c|c|c|c|c|c|c|c|c|c|c|c|}
\hline \multirow[b]{3}{*}{ Element } & \multirow[b]{3}{*}{$\lambda(\AA)$} & \multirow[b]{3}{*}{$\chi(\mathrm{eV})$} & \multirow[b]{3}{*}{$\log g f$} & \multicolumn{8}{|c|}{ Equivalent Widths (mÅ) } \\
\hline & & & & \multicolumn{8}{|c|}{ Star } \\
\hline & & & & 11029 & 13540 & 13964 & 26606 & 27955 & 28064 & 31364 & 32782 \\
\hline \multirow[t]{53}{*}{ Fe I } & 5162.27 & 4.18 & 0.079 & $\ldots$ & 153 & $\ldots$ & $\ldots$ & $\ldots$ & $\ldots$ & 143 & $\ldots$ \\
\hline & 5198.71 & 2.22 & -2.140 & $\ldots$ & $\ldots$ & $\ldots$ & $\ldots$ & $\ldots$ & $\ldots$ & 126 & $\ldots$ \\
\hline & 5242.49 & 3.63 & -0.970 & $\ldots$ & $\ldots$ & $\ldots$ & $\ldots$ & $\ldots$ & 126 & 98 & $\ldots$ \\
\hline & 5288.52 & 3.69 & -1.510 & $\ldots$ & $\ldots$ & $\ldots$ & $\ldots$ & $\ldots$ & $\ldots$ & 82 & $\ldots$ \\
\hline & 5307.36 & 1.61 & -2.970 & 160 & 140 & $\ldots$ & $\ldots$ & 149 & $\ldots$ & $\ldots$ & $\ldots$ \\
\hline & 5315.05 & 4.37 & -1.400 & 64 & 63 & $\ldots$ & $\ldots$ & $\ldots$ & $\ldots$ & $\ldots$ & $\ldots$ \\
\hline & 5321.11 & 4.43 & -1.190 & $\ldots$ & $\ldots$ & $\ldots$ & $\ldots$ & $\ldots$ & 69 & $\ldots$ & $\ldots$ \\
\hline & 5322.04 & 2.28 & -2.840 & $\ldots$ & $\ldots$ & 128 & 127 & 108 & $\ldots$ & $\ldots$ & $\ldots$ \\
\hline & 5364.87 & 4.45 & 0.230 & $\ldots$ & 153 & $\ldots$ & $\ldots$ & $\ldots$ & $\ldots$ & $\ldots$ & $\ldots$ \\
\hline & 5373.71 & 4.47 & -0.710 & 95 & 97 & 84 & $\ldots$ & $\ldots$ & $\ldots$ & 84 & $\ldots$ \\
\hline & 5389.48 & 4.42 & -0.250 & 159 & $\ldots$ & $\ldots$ & $\ldots$ & 131 & $\ldots$ & $\ldots$ & $\ldots$ \\
\hline & 5393.17 & 3.24 & -0.720 & $\ldots$ & 156 & $\ldots$ & $\ldots$ & $\ldots$ & $\ldots$ & 149 & $\ldots$ \\
\hline & 5417.03 & 4.42 & -1.530 & $\ldots$ & $\ldots$ & $\ldots$ & $\ldots$ & $\ldots$ & $\ldots$ & $\ldots$ & 53 \\
\hline & 5441.34 & 4.31 & -1.580 & 45 & $\ldots$ & $\ldots$ & $\ldots$ & $\ldots$ & 42 & $\ldots$ & $\ldots$ \\
\hline & 5445.04 & 4.39 & -0.041 & $\ldots$ & 133 & $\ldots$ & $\ldots$ & $\ldots$ & 153 & $\ldots$ & $\cdots$ \\
\hline & 5522.45 & 4.21 & -1.400 & 70 & $\ldots$ & $\ldots$ & 39 & $\ldots$ & $\ldots$ & $\ldots$ & $\ldots$ \\
\hline & 5531.98 & 4.91 & -1.460 & $\ldots$ & $\ldots$ & $\ldots$ & 59 & 20 & 30 & $\ldots$ & $\ldots$ \\
\hline & 5532.75 & 3.57 & -2.000 & $\ldots$ & $\ldots$ & 40 & $\ldots$ & $\ldots$ & $\ldots$ & $\ldots$ & $\ldots$ \\
\hline & 5554.90 & 4.55 & -0.380 & $\ldots$ & $\ldots$ & $\ldots$ & $\ldots$ & 125 & $\ldots$ & $\ldots$ & $\ldots$ \\
\hline & 5560.21 & 4.43 & -1.040 & 85 & $\ldots$ & $\ldots$ & 67 & $\ldots$ & $\ldots$ & 70 & $\ldots$ \\
\hline & 5567.39 & 2.61 & -2.560 & 102 & $\ldots$ & $\ldots$ & $\ldots$ & $\ldots$ & 124 & 83 & $\ldots$ \\
\hline & 5584.77 & 3.57 & -2.170 & $\ldots$ & $\ldots$ & $\ldots$ & $\ldots$ & $\ldots$ & $\ldots$ & 68 & $\ldots$ \\
\hline & 5624.02 & 4.39 & -1.330 & 78 & $\ldots$ & $\ldots$ & $\ldots$ & $\ldots$ & $\ldots$ & $\ldots$ & $\ldots$ \\
\hline & 5633.95 & 4.99 & -0.120 & $\ldots$ & $\ldots$ & 105 & 107 & 76 & $\ldots$ & $\ldots$ & $\ldots$ \\
\hline & 5635.82 & 4.26 & -1.740 & 56 & $\ldots$ & $\ldots$ & $\ldots$ & 25 & $\ldots$ & $\ldots$ & 40 \\
\hline & 5638.26 & 4.22 & -0.720 & 116 & $\ldots$ & $\ldots$ & $\ldots$ & $\ldots$ & $\ldots$ & $\ldots$ & 131 \\
\hline & 5686.53 & 4.55 & -0.450 & 122 & $\ldots$ & $\ldots$ & $\ldots$ & $\ldots$ & $\ldots$ & $\ldots$ & $\ldots$ \\
\hline & 5691.50 & 4.30 & -1.370 & $\ldots$ & $\ldots$ & $\ldots$ & $\ldots$ & 64 & 83 & $\ldots$ & $\ldots$ \\
\hline & 5705.47 & 4.30 & -1.360 & $\ldots$ & $\ldots$ & 82 & $\ldots$ & 49 & 67 & 51 & 76 \\
\hline & 5717.83 & 4.28 & -0.979 & $\ldots$ & $\ldots$ & $\ldots$ & 88 & 80 & $\ldots$ & $\ldots$ & $\ldots$ \\
\hline & 5731.76 & 4.26 & -1.150 & 101 & $\ldots$ & $\ldots$ & $\ldots$ & $\ldots$ & 91 & $\ldots$ & $\ldots$ \\
\hline & 5806.73 & 4.61 & -0.900 & 75 & $\ldots$ & $\ldots$ & $\ldots$ & $\ldots$ & $\ldots$ & $\ldots$ & 80 \\
\hline & 5814.81 & 4.28 & -1.820 & $\ldots$ & $\ldots$ & $\ldots$ & $\ldots$ & $\ldots$ & 37 & 29 & $\ldots$ \\
\hline & 5852.22 & 4.55 & -1.180 & 78 & 76 & $\ldots$ & $\ldots$ & $\ldots$ & $\ldots$ & 51 & $\ldots$ \\
\hline & 5883.82 & 3.96 & -1.210 & $\ldots$ & $\ldots$ & 95 & $\ldots$ & 79 & 101 & $\ldots$ & $\ldots$ \\
\hline & 5916.25 & 2.45 & -2.990 & $\ldots$ & 99 & 97 & $\ldots$ & $\ldots$ & 108 & 86 & 99 \\
\hline & 5934.65 & 3.93 & -1.020 & 111 & 103 & 105 & $\ldots$ & 113 & $\ldots$ & $\ldots$ & $\ldots$ \\
\hline & 6020.17 & 4.61 & -0.210 & $\ldots$ & $\ldots$ & $\ldots$ & $\ldots$ & 128 & $\ldots$ & $\ldots$ & $\ldots$ \\
\hline & 6024.06 & 4.55 & -0.060 & 141 & $\ldots$ & 124 & $\ldots$ & 128 & 123 & $\ldots$ & $\ldots$ \\
\hline & 6027.05 & 4.08 & -1.090 & $\ldots$ & 107 & 112 & 92 & 87 & $\ldots$ & $\ldots$ & $\ldots$ \\
\hline & 6056.01 & 4.73 & -0.400 & $\ldots$ & $\ldots$ & 92 & $\ldots$ & 99 & $\ldots$ & 80 & 127 \\
\hline & 6079.01 & 4.65 & -0.970 & 76 & $\ldots$ & 60 & $\ldots$ & $\ldots$ & $\ldots$ & $\ldots$ & $\ldots$ \\
\hline & 6082.71 & 2.22 & -3.580 & $\ldots$ & $\ldots$ & $\ldots$ & $\ldots$ & $\ldots$ & 98 & $\ldots$ & $\ldots$ \\
\hline & 6093.64 & 4.61 & -1.350 & 46 & $\ldots$ & $\ldots$ & $\ldots$ & $\ldots$ & $\ldots$ & $\ldots$ & $\ldots$ \\
\hline & 6096.66 & 3.98 & -1.780 & 70 & 65 & $\ldots$ & $\ldots$ & $\ldots$ & $\ldots$ & 65 & $\ldots$ \\
\hline & 6120.25 & 0.91 & -5.950 & $\ldots$ & $\ldots$ & $\ldots$ & $\ldots$ & $\ldots$ & 42 & $\ldots$ & $\ldots$ \\
\hline & 6151.62 & 2.18 & -3.290 & 98 & 92 & 112 & $\ldots$ & 79 & 103 & $\ldots$ & $\ldots$ \\
\hline & 6157.73 & 4.08 & -1.110 & $\ldots$ & $\ldots$ & $\ldots$ & 85 & $\ldots$ & $\ldots$ & 85 & $\ldots$ \\
\hline & 6165.36 & 4.14 & -1.470 & 64 & 66 & 75 & 93 & 78 & $\ldots$ & 71 & $\ldots$ \\
\hline & 6170.51 & 4.79 & -0.380 & $\ldots$ & 102 & $\ldots$ & $\ldots$ & $\ldots$ & $\ldots$ & $\ldots$ & $\ldots$ \\
\hline & 6173.34 & 2.22 & -2.880 & 126 & $\ldots$ & $\ldots$ & $\ldots$ & $\ldots$ & 146 & $\ldots$ & 155 \\
\hline & 6187.99 & 3.94 & -1.570 & 82 & 77 & 89 & $\ldots$ & 57 & 88 & 72 & $\ldots$ \\
\hline & 6200.31 & 2.60 & -2.440 & 130 & $\ldots$ & 133 & $\ldots$ & 118 & 144 & $\ldots$ & $\ldots$ \\
\hline \multirow[t]{9}{*}{$\mathrm{Fe} \mathrm{I}$} & 6213.43 & 2.22 & -2.480 & 149 & $\ldots$ & $\ldots$ & $\ldots$ & $\ldots$ & $\ldots$ & $\ldots$ & $\ldots$ \\
\hline & 6254.26 & 2.28 & -2.440 & 157 & $\ldots$ & $\ldots$ & $\ldots$ & $\ldots$ & $\ldots$ & $\ldots$ & $\ldots$ \\
\hline & 6265.13 & 2.18 & -2.550 & 147 & $\ldots$ & $\ldots$ & $\ldots$ & 143 & $\ldots$ & 124 & $\ldots$ \\
\hline & 6322.69 & 2.59 & -2.430 & 137 & $\ldots$ & $\ldots$ & $\ldots$ & 124 & $\ldots$ & $\ldots$ & 142 \\
\hline & 6380.74 & 4.19 & -1.320 & 86 & 84 & $\ldots$ & $\ldots$ & 83 & 81 & $\ldots$ & $\ldots$ \\
\hline & 6392.54 & 2.28 & -4.030 & 62 & $\ldots$ & $\ldots$ & $\ldots$ & 35 & 51 & $\ldots$ & $\ldots$ \\
\hline & 6411.65 & 3.65 & -0.660 & $\ldots$ & 139 & $\ldots$ & $\ldots$ & $\ldots$ & $\ldots$ & $\ldots$ & $\ldots$ \\
\hline & 6421.35 & 2.28 & -2.010 & $\ldots$ & $\ldots$ & $\ldots$ & $\ldots$ & 149 & $\ldots$ & 151 & $\ldots$ \\
\hline & 6436.41 & 4.19 & -2.460 & $\ldots$ & $\ldots$ & $\ldots$ & $\ldots$ & 20 & 10 & $\ldots$ & $\ldots$ \\
\hline
\end{tabular}


Table 6

(Continued)

\begin{tabular}{|c|c|c|c|c|c|c|c|c|c|c|c|}
\hline \multirow[b]{3}{*}{ Element } & \multirow[b]{3}{*}{$\lambda(\AA)$} & \multirow[b]{3}{*}{$\chi(\mathrm{eV})$} & \multirow[b]{3}{*}{$\log g f$} & \multicolumn{8}{|c|}{ Equivalent Widths (mÅ) } \\
\hline & & & & \multicolumn{8}{|c|}{ Star } \\
\hline & & & & 11029 & 13540 & 13964 & 26606 & 27955 & 28064 & 31364 & 32782 \\
\hline & 6469.19 & 4.83 & -0.620 & 98 & 79 & $\ldots$ & 116 & 75 & 72 & $\ldots$ & $\ldots$ \\
\hline & 6574.23 & 0.99 & -5.020 & $\ldots$ & 86 & $\ldots$ & 101 & 61 & 102 & 78 & $\ldots$ \\
\hline & 6591.31 & 4.59 & -2.070 & 20 & $\ldots$ & $\ldots$ & $\ldots$ & $\ldots$ & $\ldots$ & $\ldots$ & $\ldots$ \\
\hline & 6593.87 & 2.44 & -2.420 & $\ldots$ & $\ldots$ & $\ldots$ & $\ldots$ & 129 & $\ldots$ & $\ldots$ & $\ldots$ \\
\hline & 6597.56 & 4.79 & -0.920 & 60 & $\cdots$ & $\ldots$ & 80 & $\ldots$ & $\ldots$ & 46 & $\cdots$ \\
\hline & 6608.03 & 2.28 & -4.030 & 61 & 37 & 58 & 90 & $\cdots$ & 63 & $\ldots$ & $\ldots$ \\
\hline & 6609.11 & 2.56 & -2.690 & 122 & $\ldots$ & $\ldots$ & $\ldots$ & $\ldots$ & 110 & $\ldots$ & 133 \\
\hline & 6646.93 & 2.61 & -3.990 & $\ldots$ & 23 & $\ldots$ & $\ldots$ & 18 & 25 & $\ldots$ & $\ldots$ \\
\hline & 6653.85 & 4.14 & -2.520 & $\ldots$ & $\ldots$ & $\ldots$ & $\ldots$ & $\ldots$ & $\ldots$ & 22 & $\ldots$ \\
\hline & 6703.57 & 2.76 & -3.160 & 68 & 79 & 78 & 53 & 41 & $\ldots$ & $\ldots$ & 55 \\
\hline & 6710.32 & 1.80 & -4.880 & $\ldots$ & $\ldots$ & $\ldots$ & 68 & $\ldots$ & $\ldots$ & $\ldots$ & $\ldots$ \\
\hline & 6739.52 & 1.56 & -4.950 & 39 & $\ldots$ & 57 & 86 & $\ldots$ & 58 & 34 & 59 \\
\hline & 6750.15 & 2.42 & -2.620 & 118 & $\ldots$ & $\ldots$ & 147 & $\ldots$ & $\ldots$ & 118 & 148 \\
\hline & 6752.71 & 4.64 & -1.200 & 59 & 47 & $\ldots$ & $\ldots$ & 45 & $\ldots$ & 60 & $\ldots$ \\
\hline & 6806.85 & 2.73 & -3.210 & 77 & $\ldots$ & 97 & 113 & $\ldots$ & 83 & $\ldots$ & $\ldots$ \\
\hline & 6820.37 & 4.64 & -1.170 & $\ldots$ & 74 & $\ldots$ & $\ldots$ & $\cdots$ & 43 & 50 & $\cdots$ \\
\hline & 6841.34 & 4.61 & -0.600 & 124 & $\ldots$ & $\ldots$ & $\ldots$ & $\ldots$ & $\ldots$ & $\ldots$ & $\ldots$ \\
\hline & 6851.64 & 1.61 & -5.320 & $\ldots$ & $\ldots$ & $\ldots$ & 52 & $\ldots$ & $\ldots$ & 24 & $\ldots$ \\
\hline & 6858.15 & 4.61 & -0.930 & $\ldots$ & 91 & 75 & $\ldots$ & 88 & 81 & $\ldots$ & 64 \\
\hline & 7130.92 & 4.22 & -0.700 & 129 & 126 & 126 & $\ldots$ & 111 & 115 & 113 & $\ldots$ \\
\hline & 7132.99 & 4.08 & -1.610 & $\ldots$ & $\ldots$ & 76 & $\ldots$ & $\ldots$ & $\ldots$ & 66 & $\ldots$ \\
\hline \multirow[t]{11}{*}{ Fe II } & 5132.66 & 2.81 & -4.000 & $\ldots$ & $\ldots$ & $\ldots$ & $\ldots$ & 26 & $\ldots$ & $\ldots$ & $\ldots$ \\
\hline & 5234.62 & 3.22 & -2.240 & $\ldots$ & $\ldots$ & $\ldots$ & $\ldots$ & 108 & $\ldots$ & $\ldots$ & $\ldots$ \\
\hline & 5425.25 & 3.20 & -3.210 & 60 & 70 & 74 & 39 & 44 & $\ldots$ & 75 & 59 \\
\hline & 5534.83 & 3.25 & -2.770 & 95 & $\ldots$ & $\ldots$ & $\ldots$ & 76 & $\ldots$ & $\ldots$ & $\ldots$ \\
\hline & 5991.37 & 3.15 & -3.560 & 66 & $\ldots$ & $\ldots$ & $\ldots$ & $\ldots$ & $\ldots$ & $\ldots$ & $\ldots$ \\
\hline & 6084.10 & 3.20 & -3.800 & 39 & $\ldots$ & $\ldots$ & $\ldots$ & 17 & 26 & $\ldots$ & $\ldots$ \\
\hline & 6149.25 & 3.89 & -2.720 & 53 & $\ldots$ & 49 & 45 & $\cdots$ & 42 & 70 & $\cdots$ \\
\hline & 6247.55 & 3.89 & -2.340 & 82 & 86 & 56 & $\ldots$ & $\ldots$ & 78 & 81 & $\ldots$ \\
\hline & 6369.46 & 2.89 & -4.110 & $\ldots$ & $\ldots$ & $\ldots$ & $\ldots$ & $\ldots$ & 43 & $\ldots$ & $\ldots$ \\
\hline & 6416.92 & 3.89 & -2.680 & 63 & 67 & $\ldots$ & $\ldots$ & 43 & 41 & $\ldots$ & $\ldots$ \\
\hline & 6432.68 & 2.89 & -3.580 & 61 & $\ldots$ & $\ldots$ & 36 & $\ldots$ & $\ldots$ & $\ldots$ & $\ldots$ \\
\hline
\end{tabular}

their associated errors were obtained from the average and error-of-the-mean of these measurements.

\subsection{Reddening and Distances}

The reddening for the stars of box B was estimated using the isochrones of Bertelli et al. $(2008,2009)$ to obtain the $(V-I)$ intrinsic color of each star. In Table 3 we show the $E(V-I)$ values obtained for the stars of box B. We also calculated the distances for each star of box B using the equation

$$
\begin{aligned}
\log d(\mathrm{pc})= & 1 / 2\left[\log \frac{M_{\star}}{M_{\odot}}+0.4\left(V-A_{V}+\mathrm{BC}\right)\right) \\
& \left.\left.+4 \log T_{\text {eff }}-\log g_{\star}-10.62\right],\right]
\end{aligned}
$$

where $T_{\text {eff }}$ and $\log g_{\star}$ are the effective temperature and surface gravity, respectively, and $M$ is the mass obtained through the evolutionary tracks of Bertelli et al. (2008, 2009). The photometric data of Table 2 were combined with bolometric corrections defined by the relations in Alonso et al. (1999). The extinction in $V\left(A_{V}\right)$ for each star was calculated using the reddening $E(V-I)$ shown in Table 3, with the nonstandard absorption law valid for the third Galactic quadrant, where $R_{V}=2.9$ and $E(B-V)=0.7955 \times E(V-I)$ (Turner et al. 2011a). For the Sun, we adopted $M_{\mathrm{bol} \odot}=4.74 \mathrm{mag}$ (Bessell et al. 1998), $T_{\text {eff } \odot}=5700 \mathrm{~K}$, and $\log g_{\odot}=4.3$ dex.

We also obtained a rough estimate of the age of the stars from box B using the spectroscopic atmospheric parameters and isochrones of Bertelli et al. (2008, 2009). We note that such a sample is composed of a great mix of stars with age ranging from $1.2 \mathrm{Myr}$ to $10 \mathrm{Gyr}$. This huge range is not unexpected with a sample containing both thin- and thick-disk stars.

The reddening and distance of box C and D stars were similarly derived. The intrinsic colors and absolute magnitudes of box $\mathrm{C}$ stars were derived from comparison of their position in the temperature-gravity plane with the same solarmetallicity isochrones used for box B. The intrinsic color, compared with the observed color, returned the reddening $E(V-I)$, which was used to derive $A_{V}$ with the same equations given above. As we had no gravity information for box D stars and argued that they are all MS objects, we adopted for them the absolute magnitude of solar-metallicity ZAMS objects at the corresponding temperature. From the derived absolute magnitude, observed $V$ magnitude, and $A_{V}$, the distances were straightforwardly computed. We chose to use $(V-I)$ as the temperature indicator for consistency with what was done in box B. However, a bluer color could be a better 
Table 7

Other Lines Studied

\begin{tabular}{|c|c|c|c|c|c|c|c|c|c|c|c|c|}
\hline \multirow[b]{3}{*}{ Element } & \multirow[b]{3}{*}{$\lambda$} & \multirow[b]{3}{*}{$\chi(\mathrm{eV})$} & \multirow[b]{3}{*}{$\log g f$} & \multirow[b]{3}{*}{ Ref } & \multicolumn{8}{|c|}{ Equivalent Widths (mÅ) } \\
\hline & & & & & \multicolumn{8}{|c|}{ Star } \\
\hline & & & & & 11029 & 13540 & 13964 & 26606 & 27955 & 28064 & 31364 & 32782 \\
\hline $\mathrm{Na} I$ & 6154.22 & 2.10 & -1.51 & PS & 73 & 79 & $\cdots$ & $\cdots$ & $\cdots$ & 66 & 59 & 88 \\
\hline $\mathrm{Na} \mathrm{I}$ & 6160.75 & 2.10 & -1.21 & R03 & $\cdots$ & 99 & 89 & $\ldots$ & $\ldots$ & $\ldots$ & $\cdots$ & $\cdots$ \\
\hline Mg I & 4730.04 & 4.34 & -2.39 & $\mathrm{R} 03$ & 81 & $\cdots$ & $\ldots$ & $\ldots$ & $\ldots$ & $\ldots$ & $\ldots$ & $\ldots$ \\
\hline Mg I & 5711.10 & 4.34 & -1.75 & R99 & 146 & $\cdots$ & $\ldots$ & $\ldots$ & 127 & $\ldots$ & $\ldots$ & $\ldots$ \\
\hline Mg I & 7387.70 & 5.75 & -0.87 & MR94 & $\ldots$ & $\cdots$ & 115 & 120 & 77 & $\ldots$ & 54 & $\cdots$ \\
\hline $\operatorname{Mg} \mathrm{I}$ & 8717.83 & 5.91 & -0.97 & WSM & $\cdots$ & $\cdots$ & $\cdots$ & 88 & $\cdots$ & 70 & $\cdots$ & 129 \\
\hline Mg I & 8736.04 & 5.94 & -0.34 & WSM & $\ldots$ & $\ldots$ & $\ldots$ & $\ldots$ & $\ldots$ & 112 & 91 & $\ldots$ \\
\hline $\mathrm{Al} \mathrm{I}$ & 6698.67 & 3.14 & -1.63 & R03 & $\ldots$ & $\ldots$ & $\ldots$ & 74 & $\ldots$ & $\ldots$ & $\ldots$ & $\ldots$ \\
\hline $\mathrm{Al} \mathrm{I}$ & 7835.32 & 4.04 & -0.58 & R03 & $\cdots$ & 62 & 45 & $\ldots$ & $\ldots$ & 32 & $\ldots$ & 82 \\
\hline $\mathrm{Al} \mathrm{I}$ & 7836.13 & 4.02 & -0.40 & R03 & $\ldots$ & 72 & 59 & $\ldots$ & $\ldots$ & 54 & $\ldots$ & $\ldots$ \\
\hline $\mathrm{Al} \mathrm{I}$ & 8772.88 & 4.02 & -0.25 & R03 & $\ldots$ & $\ldots$ & $\ldots$ & $\ldots$ & $\ldots$ & 66 & $\ldots$ & $\ldots$ \\
\hline $\mathrm{Al} \mathrm{I}$ & 8773.91 & 4.02 & -0.07 & R03 & $\ldots$ & $\ldots$ & $\ldots$ & $\ldots$ & $\ldots$ & 65 & $\ldots$ & $\ldots$ \\
\hline $\mathrm{Si} \mathrm{I}$ & 5793.08 & 4.93 & -2.06 & R03 & $\ldots$ & 76 & $\ldots$ & $\ldots$ & 51 & $\ldots$ & $\ldots$ & $\ldots$ \\
\hline Si I & 6125.03 & 5.61 & -1.54 & E93 & 55 & 56 & $\ldots$ & $\ldots$ & $\ldots$ & $\ldots$ & 40 & $\cdots$ \\
\hline $\mathrm{Si} \mathrm{I}$ & 6131.58 & 5.62 & -1.69 & E93 & $\ldots$ & $\ldots$ & $\ldots$ & $\ldots$ & $\ldots$ & $\ldots$ & 45 & $\ldots$ \\
\hline Si I & 6145.02 & 5.61 & -1.43 & E93 & 58 & $\ldots$ & $\ldots$ & $\ldots$ & $\ldots$ & $\ldots$ & $\ldots$ & $\ldots$ \\
\hline Si I & 6155.14 & 5.62 & -0.77 & E93 & $\ldots$ & 95 & $\ldots$ & $\ldots$ & 86 & $\ldots$ & 79 & $\ldots$ \\
\hline Si I & 8728.01 & 6.18 & -0.36 & E93 & $\ldots$ & $\ldots$ & $\ldots$ & 97 & $\ldots$ & $\ldots$ & $\ldots$ & $\ldots$ \\
\hline $\mathrm{Si} \mathrm{I}$ & 8742.45 & 5.87 & -0.51 & E93 & $\ldots$ & $\ldots$ & $\ldots$ & $\ldots$ & $\ldots$ & 86 & $\ldots$ & $\ldots$ \\
\hline $\mathrm{Ca} \mathrm{I}$ & 6161.30 & 2.52 & -1.27 & E93 & $\ldots$ & 107 & 125 & $\ldots$ & $\ldots$ & $\ldots$ & $\ldots$ & $\ldots$ \\
\hline $\mathrm{Ca} \mathrm{I}$ & 6166.44 & 2.52 & -1.14 & R03 & 87 & 112 & 140 & $\ldots$ & 101 & $\ldots$ & 89 & $\ldots$ \\
\hline $\mathrm{Ca} \mathrm{I}$ & 6169.04 & 2.52 & -0.80 & R03 & 129 & $\ldots$ & 154 & $\ldots$ & $\ldots$ & $\ldots$ & $\ldots$ & $\ldots$ \\
\hline $\mathrm{Ca} \mathrm{I}$ & 6169.56 & 2.53 & -0.48 & DS91 & 142 & $\ldots$ & $\ldots$ & $\ldots$ & $\ldots$ & $\ldots$ & $\ldots$ & $\ldots$ \\
\hline $\mathrm{Ca} \mathrm{I}$ & 6455.60 & 2.51 & -1.29 & R03 & 78 & $\ldots$ & 121 & $\ldots$ & 64 & $\ldots$ & $\ldots$ & $\ldots$ \\
\hline Ca I & 6471.66 & 2.51 & -0.69 & S86 & $\ldots$ & 132 & $\ldots$ & $\ldots$ & 115 & 117 & 106 & $\ldots$ \\
\hline Ti I & 4758.12 & 2.25 & 0.420 & MFK & $\cdots$ & 92 & $\ldots$ & 125 & $\cdots$ & $\ldots$ & $\ldots$ & $\cdots$ \\
\hline Ti I & 5039.96 & 0.02 & -1.130 & MFK & $\ldots$ & $\ldots$ & $\ldots$ & $\ldots$ & 148 & $\ldots$ & $\ldots$ & $\cdots$ \\
\hline Ti I & 5043.59 & 0.84 & -1.733 & MFK & 63 & $\ldots$ & $\ldots$ & $\ldots$ & $\ldots$ & $\ldots$ & $\ldots$ & $\ldots$ \\
\hline Ti I & 5062.10 & 2.16 & -0.464 & MFK & $\ldots$ & $\ldots$ & $\ldots$ & $\ldots$ & 50 & $\ldots$ & $\ldots$ & $\ldots$ \\
\hline Ti I & 5113.45 & 1.44 & -0.880 & E93 & $\ldots$ & $\ldots$ & $\ldots$ & $\ldots$ & $\ldots$ & 93 & $\ldots$ & $\ldots$ \\
\hline Ti I & 5223.63 & 2.09 & -0.559 & MFK & $\ldots$ & $\ldots$ & 50 & $\ldots$ & $\ldots$ & $\ldots$ & $\ldots$ & $\ldots$ \\
\hline Ti I & 5295.78 & 1.05 & -1.633 & MFK & $\ldots$ & $\ldots$ & $\ldots$ & $\ldots$ & $\ldots$ & 72 & $\ldots$ & $\ldots$ \\
\hline Ti I & 5490.16 & 1.46 & -0.937 & MFK & $\cdots$ & 69 & $\ldots$ & $\ldots$ & $\cdots$ & $\ldots$ & $\cdots$ & $\cdots$ \\
\hline Ti I & 5689.48 & 2.30 & -0.469 & MFK & $\ldots$ & $\ldots$ & $\ldots$ & $\ldots$ & $\ldots$ & 46 & $\ldots$ & 64 \\
\hline Ti I & 5866.46 & 1.07 & -0.871 & E93 & 106 & $\ldots$ & $\ldots$ & $\ldots$ & 109 & $\ldots$ & 95 & $\ldots$ \\
\hline Ti I & 5922.12 & 1.05 & -1.465 & MFK & $\ldots$ & $\cdots$ & 83 & $\ldots$ & $\ldots$ & $\ldots$ & 76 & $\ldots$ \\
\hline Ti I & 5978.55 & 1.87 & -0.496 & MFK & 81 & 75 & $\ldots$ & $\ldots$ & 75 & $\ldots$ & $\ldots$ & $\ldots$ \\
\hline Ti I & 6091.18 & 2.27 & -0.370 & R03 & 67 & $\ldots$ & $\ldots$ & $\ldots$ & $\ldots$ & $\ldots$ & 42 & $\ldots$ \\
\hline Ti I & 6126.22 & 1.07 & -1.370 & R03 & 84 & $\ldots$ & 103 & 128 & $\ldots$ & 81 & $\ldots$ & 101 \\
\hline Ti I & 6258.11 & 1.44 & -0.355 & MFK & $\ldots$ & $\ldots$ & 118 & $\ldots$ & $\ldots$ & $\ldots$ & $\ldots$ & $\ldots$ \\
\hline Ti I & 6261.11 & 1.43 & -0.480 & B86 & $\ldots$ & $\ldots$ & 127 & $\ldots$ & 105 & $\ldots$ & $\ldots$ & $\ldots$ \\
\hline Ti I & 6554.24 & 1.44 & -1.219 & MFK & $\ldots$ & $\ldots$ & 49 & $\ldots$ & $\ldots$ & $\ldots$ & $\ldots$ & 100 \\
\hline Cr I & 5193.50 & 3.42 & -0.720 & MFK & 27 & $\ldots$ & $\ldots$ & $\ldots$ & $\ldots$ & $\ldots$ & $\cdots$ & $\ldots$ \\
\hline Cr I & 5214.13 & 3.37 & -0.740 & MFK & $\ldots$ & $\ldots$ & $\ldots$ & $\ldots$ & 20 & $\ldots$ & $\ldots$ & $\ldots$ \\
\hline Cr I & 5296.70 & 0.98 & -1.390 & GS & 154 & $\ldots$ & $\ldots$ & $\cdots$ & $\cdots$ & $\ldots$ & $\cdots$ & $\cdots$ \\
\hline Cr I & 5304.18 & 3.46 & -0.692 & MFK & $\ldots$ & $\ldots$ & $\ldots$ & 42 & $\ldots$ & $\ldots$ & $\ldots$ & $\ldots$ \\
\hline Cr I & 5345.81 & 1.00 & -0.980 & MFK & $\ldots$ & $\ldots$ & $\ldots$ & $\ldots$ & $\ldots$ & $\ldots$ & 151 & $\ldots$ \\
\hline Cr I & 5348.32 & 1.00 & -1.290 & GS & 158 & 132 & $\ldots$ & $\ldots$ & 144 & $\ldots$ & $\ldots$ & $\ldots$ \\
\hline Cr I & 5783.07 & 3.32 & -0.500 & MFK & $\ldots$ & $\ldots$ & 64 & $\ldots$ & 47 & 73 & $\ldots$ & $\ldots$ \\
\hline $\mathrm{Cr} \mathrm{I}$ & 5787.93 & 3.32 & -0.080 & GS & $\ldots$ & 58 & $\ldots$ & $\ldots$ & $\ldots$ & 75 & $\ldots$ & $\ldots$ \\
\hline Cr I & 6330.09 & 0.94 & -2.920 & R03 & $\ldots$ & 57 & $\ldots$ & $\ldots$ & $\ldots$ & $\cdots$ & 65 & 85 \\
\hline $\mathrm{Ni} \mathrm{I}$ & 4904.42 & 3.54 & -0.170 & MFK & $\ldots$ & 125 & $\ldots$ & $\ldots$ & 143 & $\ldots$ & $\ldots$ & 136 \\
\hline $\mathrm{Ni} I$ & 4935.83 & 3.94 & -0.360 & MFK & $\ldots$ & 83 & $\ldots$ & $\ldots$ & 88 & 70 & $\ldots$ & $\ldots$ \\
\hline $\mathrm{Ni} I$ & 4953.21 & 3.74 & -0.660 & MFK & $\ldots$ & $\ldots$ & $\ldots$ & $\ldots$ & 88 & $\ldots$ & $\ldots$ & $\ldots$ \\
\hline $\mathrm{Ni}$ I & 5010.94 & 3.63 & -0.870 & MFK & $\ldots$ & $\ldots$ & 87 & $\ldots$ & $\cdots$ & $\ldots$ & $\ldots$ & $\cdots$ \\
\hline $\mathrm{Ni} I$ & 5578.73 & 1.68 & -2.640 & MFK & 108 & $\ldots$ & $\ldots$ & $\ldots$ & $\ldots$ & 110 & 90 & $\ldots$ \\
\hline $\mathrm{Ni} I$ & 5593.75 & 3.90 & -0.840 & MFK & 60 & $\ldots$ & $\ldots$ & $\ldots$ & 73 & 45 & $\ldots$ & 85 \\
\hline $\mathrm{Ni} I$ & 5643.09 & 4.17 & -1.250 & MFK & 24 & 40 & $\ldots$ & $\ldots$ & $\cdots$ & $\cdots$ & $\ldots$ & $\cdots$ \\
\hline $\mathrm{Ni}$ I & 5748.36 & 1.68 & -3.260 & MFK & $\ldots$ & $\ldots$ & $\ldots$ & $\ldots$ & $\ldots$ & $\ldots$ & $\ldots$ & 73 \\
\hline $\mathrm{Ni}$ I & 5805.23 & 4.17 & -0.640 & MFK & $\ldots$ & $\cdots$ & $\ldots$ & $\ldots$ & 72 & 43 & $\ldots$ & $\ldots$ \\
\hline $\mathrm{Ni} I$ & 6053.69 & 4.24 & -1.070 & MFK & $\ldots$ & 49 & $\ldots$ & $\cdots$ & $\ldots$ & $\ldots$ & $\cdots$ & $\cdots$ \\
\hline $\mathrm{Ni} I$ & 6086.29 & 4.27 & -0.510 & MFK & 62 & 81 & $\ldots$ & $\ldots$ & $\ldots$ & 50 & $\ldots$ & $\ldots$ \\
\hline
\end{tabular}


Table 7

(Continued)

\begin{tabular}{|c|c|c|c|c|c|c|c|c|c|c|c|c|}
\hline \multirow[b]{3}{*}{ Element } & \multirow[b]{3}{*}{$\lambda$} & \multirow[b]{3}{*}{$\chi(\mathrm{eV})$} & \multirow[b]{3}{*}{$\log g f$} & \multirow[b]{3}{*}{ Ref } & \multicolumn{8}{|c|}{ Equivalent Widths (mÅ) } \\
\hline & & & & & \multicolumn{8}{|c|}{ Star } \\
\hline & & & & & 11029 & 13540 & 13964 & 26606 & 27955 & 28064 & 31364 & 32782 \\
\hline $\mathrm{Ni} I$ & 6128.98 & 1.68 & -3.320 & MFK & $\ldots$ & 78 & $\ldots$ & $\ldots$ & 64 & 67 & 57 & $\ldots$ \\
\hline $\mathrm{Ni}$ I & 6176.82 & 4.09 & -0.264 & R03 & $\ldots$ & $\ldots$ & $\ldots$ & $\ldots$ & 93 & $\ldots$ & $\ldots$ & $\ldots$ \\
\hline Ni I & 6186.72 & 4.11 & -0.960 & MFK & 56 & $\ldots$ & 47 & $\ldots$ & $\cdots$ & $\ldots$ & 50 & $\ldots$ \\
\hline $\mathrm{Ni} I$ & 6204.61 & 4.09 & -1.150 & MFK & 42 & $\ldots$ & $\ldots$ & $\ldots$ & $\ldots$ & $\ldots$ & $\ldots$ & $\ldots$ \\
\hline Ni I & 6223.99 & 4.11 & -0.980 & MFK & $\ldots$ & $\ldots$ & 57 & $\ldots$ & $\ldots$ & $\ldots$ & $\ldots$ & $\ldots$ \\
\hline $\mathrm{Ni}$ I & 6230.10 & 4.11 & -1.260 & MFK & $\ldots$ & $\ldots$ & 44 & $\ldots$ & $\ldots$ & $\ldots$ & 33 & $\ldots$ \\
\hline Ni I & 6327.60 & 1.68 & -3.150 & MFK & $\ldots$ & $\ldots$ & $\ldots$ & $\ldots$ & 85 & $\ldots$ & 63 & $\ldots$ \\
\hline Ni I & 6482.81 & 1.94 & -2.630 & MFK & $\ldots$ & $\ldots$ & $\ldots$ & $\ldots$ & 83 & $\ldots$ & $\ldots$ & 100 \\
\hline $\mathrm{Ni} I$ & 6532.88 & 1.94 & -3.390 & MFK & $\ldots$ & $\ldots$ & $\ldots$ & $\ldots$ & 57 & 44 & $\ldots$ & $\ldots$ \\
\hline $\mathrm{Ni} I$ & 6586.32 & 1.95 & -2.810 & MFK & $\ldots$ & 84 & $\ldots$ & $\ldots$ & $\ldots$ & $\ldots$ & $\ldots$ & 95 \\
\hline $\mathrm{Ni} I$ & 6635.14 & 4.42 & -0.830 & MFK & $\ldots$ & 41 & $\ldots$ & $\ldots$ & $\ldots$ & $\ldots$ & 40 & $\ldots$ \\
\hline Ni I & 6643.64 & 1.68 & -2.030 & MFK & 153 & 144 & $\ldots$ & $\ldots$ & $\ldots$ & $\ldots$ & 129 & $\ldots$ \\
\hline $\mathrm{Ni} I$ & 6767.78 & 1.83 & -2.170 & MFK & 141 & $\ldots$ & $\ldots$ & $\ldots$ & $\ldots$ & $\ldots$ & 105 & $\ldots$ \\
\hline Ni I & 6772.32 & 3.66 & -0.970 & $\mathrm{R} 03$ & 73 & $\ldots$ & $\ldots$ & 83 & $\ldots$ & 84 & $\ldots$ & $\ldots$ \\
\hline Ni I & 7788.93 & 1.95 & -1.990 & E93 & 151 & 140 & $\ldots$ & $\ldots$ & $\ldots$ & $\ldots$ & 130 & $\ldots$ \\
\hline
\end{tabular}

References. B86: Blackwell et al. (1986); Ca07: Carretta et al. (2007); D2002: Depagne et al. (2002); DS91: Drake \& Smith (1991); E93: Edvardsson et al. (1993); GS: Gratton \& Sneden (1988); MFK: Martin et al. (2002); MR94: McWilliam \& Rich (1994); PS: Preston \& Sneden (2001); R03: Reddy et al. (2003); R99: Reddy et al. (1999); WSM: Wiese et al. (1969).

Table 8

Adopted Solar Abundances

\begin{tabular}{lccc}
\hline \hline Element & This Work & Grevesse \& Sauval (1998) & Asplund et al. (2009) \\
\hline $\mathrm{Fe}$ & 7.50 & 7.50 & 7.50 \\
$\mathrm{Na}$ & 6.26 & 6.33 & 6.24 \\
$\mathrm{Mg}$ & 7.55 & 7.58 & 7.60 \\
$\mathrm{Al}$ & 6.31 & 6.47 & 6.45 \\
$\mathrm{Si}$ & 7.61 & 7.55 & 7.51 \\
$\mathrm{Ca}$ & 6.37 & 6.36 & 6.34 \\
$\mathrm{Ti}$ & 4.93 & 5.02 & 4.95 \\
$\mathrm{Cr}$ & 5.65 & 5.67 & 5.64 \\
$\mathrm{Ni}$ & 6.29 & 6.25 & 6.22 \\
\hline
\end{tabular}

choice for box $\mathrm{C}$, where the stars are noticeably hotter than those in the other two groups. To test if our choice would alter the results, we repeated the procedure using $(B-V)$ instead of $(V-I)$ in box $C$. The reddening values thus derived are compatible within the errors with those previously obtained, with a mean difference and standard deviation of $-0.04 \pm 0.05 \mathrm{mag}$ and no clear trend with temperature.

\section{Results of Abundance Analysis}

Chemical abundance is one of the main pillars in characterizing stellar populations, and spectral analysis is the most reliable technique for obtaining a star's chemistry. The chemical pattern in stars allows one, for example, to distinguish which stars are from the thick disk and which ones from the thin disk (e.g., Masseron \& Gilmore 2015) and even to determine if a cluster (or star) has extragalactic origins (e.g., Sbordone et al. 2015). In this section, we present the results of our chemical analysis of the red clump stars in order to characterize this stellar population.

\subsection{Metallicity and Iron-peak Elements}

Red clump stars have been widely used to characterize the structure of the Galaxy (e.g., Lee et al. 2015; Romero-Gómez et al. 2015) mainly because they are bright enough and numerous (Bienaymé et al. 2014; Wan et al. 2015). In Galactic clusters, such stars are characterized by a similar chemical abundance, unlike the field red clump stars, which present a larger dispersion.

In Table 3, we show the metallicity derived for eight red clump stars from box B. We found that our sample of field red clump stars covers a metallicity range of $-0.68 \leqslant$ $[\mathrm{Fe} / \mathrm{H}] \leqslant 0.03$. Despite the wide dispersion in metallicity of our red clump stars, some of them have similar characteristics. Two of the target stars with the lowest metallicity (stars \#13964 and \#28064) present high estimates for the distances (20 and $12 \mathrm{kpc}$, respectively), although our results for star \#13964 are affected by a large uncertainty in atmospheric parameters and distance due to the low $\mathrm{S} / \mathrm{N}$ of its spectrum (see Table 3). Despite its higher metallicity ([Fe/ $\mathrm{H}]=-0.24)$, target \#31364 also exhibits a very large distance, $d \approx 23 \mathrm{kpc}$. We also noted that these three very distant stars have slightly similar radial velocities $(73.9,68.0$, and $65.6 \mathrm{~km} \mathrm{~s}^{-1}$ ). For stars \#11029, \#13540, \#27955, and \#32782, our results for the metallicity and distance indicate that the stars are located either near the closest edge of the outer disk or in the transition region between the outer and inner disks $\left(9 \leqslant R_{\mathrm{GC}} \leqslant 13 \mathrm{kpc}\right)$, where a large scatter of metallicity is found (Magrini et al. 2009).

$\mathrm{SNe}$ Ia are the main sources of enrichment of the interstellar medium with $\mathrm{Fe}$ and iron-peak elements, like $\mathrm{Cr}$ and $\mathrm{Ni}$. Therefore, the chemical study of iron-peak elements is important to analyze the SNe Ia production rate for the formation of the observed stellar population, as a key parameter to set, for example, the initial mass function. For our sample of red clump stars, the ranges of the abundance ratios of $[\mathrm{X} / \mathrm{Fe}]$ for $\mathrm{Cr}$ and $\mathrm{Ni}$ are $-0.09 \leqslant[\mathrm{Cr} / \mathrm{Fe}] \leqslant+0.35$ and $-0.22 \leqslant[\mathrm{Ni} / \mathrm{Fe}] \leqslant+0.24$. In Figures 8 and 9 we show the abundance ratio of $[\mathrm{X} / \mathrm{Fe}]$ for our sample of red clump field stars, for field dwarfs from Bensby et al. (2014), for disk Cepheids from Lemasle et al. (2013) and 


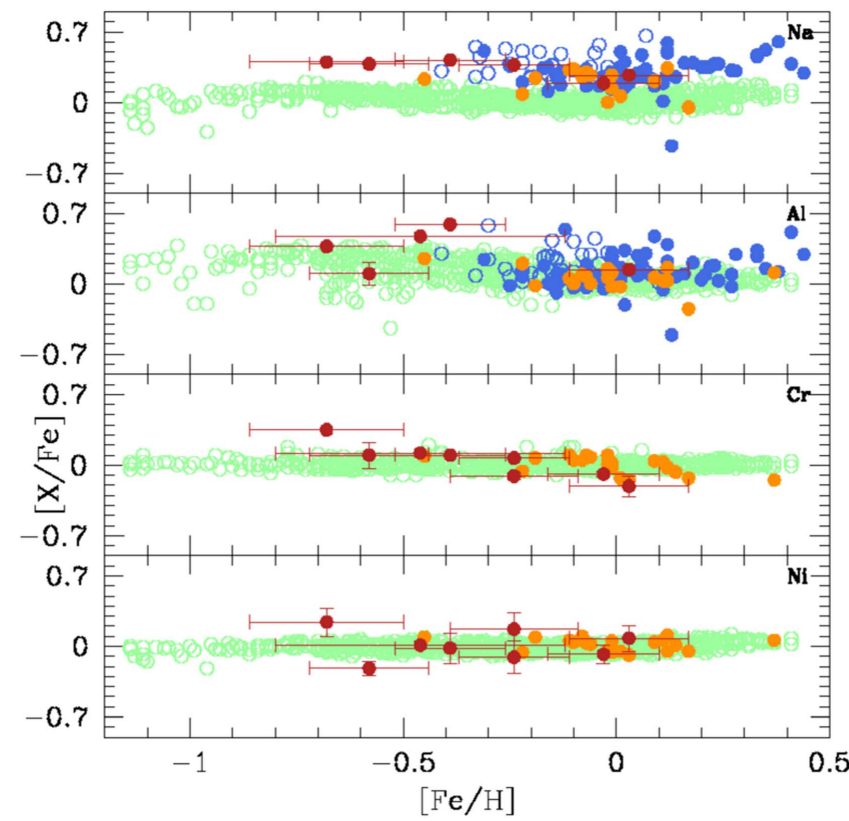

Figure 8. Abundance ratios $[\mathrm{X} / \mathrm{Fe}]$ vs. $[\mathrm{Fe} / \mathrm{H}]$. Light green open circles: field dwarfs of Bensby et al. (2014); blue open circles: Cepheids of disk of Lemasle et al. (2013); blue filled circles: Cepheids of disk of Genovali et al. (2015); red filled circles: our sample of red clump field stars; orange filled circles: open clusters from literature (Tombaugh 1 of Sales Silva et al. 2016); NGC 6192, NGC 6404, and NGC 6583 of Magrini et al. (2010); NGC 3114 of Katime Santrich et al. (2013); NGC 2527, NGC 2682, NGC 2482, NGC 2539, NGC 2335, NGC 2251, and NGC 2266 of Reddy et al. (2013); NGC 4337 of Carraro et al. (2014); Trumpler 20 of Carraro et al. (2014); NGC 4815 and NGC 6705 of Magrini et al. (2014); Cr 110, Cr 261, NGC 2477, NGC 2506, and NGC 5822 of Mishenina et al. (2015).

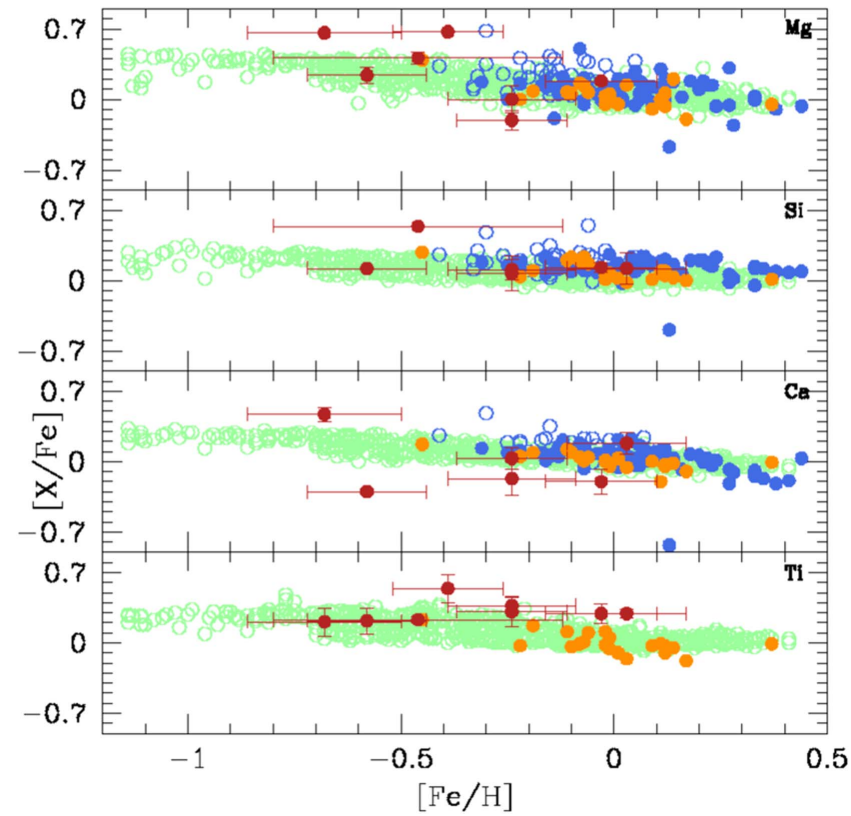

Figure 9. Abundance ratios $[\mathrm{X} / \mathrm{Fe}]$ vs. $[\mathrm{Fe} / \mathrm{H}]$. The symbols have the same meaning as in Figure 8.

Genovali et al. (2015), and also for open clusters, as described in the figure caption.

The $[\mathrm{Cr} / \mathrm{Fe}]$ and $[\mathrm{Ni} / \mathrm{Fe}]$ ratios are close to solar for all our red clump targets, as observed among disk field dwarfs and open clusters from literature in the range $-1 \leqslant[\mathrm{Fe} / \mathrm{H}] \leqslant 0$ (see Figure 8 ). The abundance ratio of nickel in the Milky
Way, in particular, is locked to the solar value at any metallicity (e.g., Sneden et al. 2004). This is usually assumed as evidence that nickel is synthesized in the same astrophysical sites as iron and in constant proportion to it. However, Sbordone et al. (2007) found that the Sagittarius dwarf spheroidal galaxy ( $\mathrm{Sgr} \mathrm{dSph}$ ) is depleted of nickel by $\approx 0.3$ dex in the whole range of $-1 \leqslant[\mathrm{Fe} / \mathrm{H}] \leqslant 0$, a behavior that could extend even down to $[\mathrm{Fe} / \mathrm{H}]=-2$ (Sbordone et al. 2015). This exotic chemical composition was, however, not observed among metal-poor $([\mathrm{Fe} / \mathrm{H}] \leqslant-2)$ globular clusters in Fornax (Letarte et al. 2006), so it is not clear if a lower $[\mathrm{Ni} / \mathrm{Fe}]$ should be expected for all dwarf galaxies at any metallicity. In any case, we find no evidence of an exotic, potentially extragalactic abundance of nickel in our sample.

\section{2. $\mathrm{Na}, \mathrm{Al}$, and $\mathrm{\alpha}$-elements}

The chemical abundances of $\alpha$-elements are constantly used to reveal the history of star formation and define the structure of the Galactic disk. In the disk, stars with a high abundance of $\alpha$ elements are associated with the thick disk, while stars with solar ratios are usually classified as belonging to the thin disk (e.g., Bensby et al. 2005). The separation of the disk into two components is usually interpreted as evidence that the stars of the thick disk had a rapid star formation with $\mathrm{SNe}$ II contributing more to the chemical enrichment of the interstellar medium than stars in the thin disk. In Table 4 we present the abundance ratios for the alpha-elements $\mathrm{Mg}, \mathrm{Si}, \mathrm{Ca}$, and $\mathrm{Ti}$ for the eight field red clump stars. We note that the $[\mathrm{X} / \mathrm{Fe}]$ ratios for $\mathrm{Ti}$ and $\mathrm{Si}$ are supersolar for our sample of red clump stars. For $\mathrm{Mg}$, the $[\mathrm{X} / \mathrm{Fe}]$ ratios show subsolar or solar values for two stars (\#27955 and \#31364), while for the other red clump stars, we got supersolar values. Finally, the $[\mathrm{Ca} / \mathrm{Fe}]$ ratio shows supersolar values for three red clump stars and subsolar values for another three stars (\#11029, \#27955, and \#2806, with values of $-0.20,-0.17$, and -0.30 , respectively).

On average, stars \#13540, \#13964, \#26606, and \#32782 have high $\alpha$-element abundances, with $[\alpha / \mathrm{Fe}]$ ratios of $0.20 \pm 0.09, \quad 0.45 \pm 0.23, \quad 0.39 \pm 0.16$, and $0.60 \pm 0.09$, respectively. Therefore, these stars are probably members of the thick disk. The other red clump stars in our sample (ID $1102,27955,28064$, and 31364) present $[\alpha / \mathrm{Fe}]$ between 0.05 and 0.10 dex and therefore probably belong to the Galactic thin disk population.

Yong et al. (2006) and Carney et al. (2005) found that field and cluster stars in the outer Galactic disk show enhancements for alpha-elements, $[\alpha / \mathrm{Fe}] \sim 0.2$, and a metallicity of approximately -0.5 dex. However, in a more recent study of giant stars in the field of the outer disk, Bensby et al. (2011) detected a thin disk of stars with $[\alpha / \mathrm{Fe}] \sim 0.0$ and a lack of stars with the chemical pattern of the thick disk $([\alpha / \mathrm{Fe}] \geqslant 0.2)$, even far above the Galactic plane. Bensby et al. (2011) concluded that this lack of thick-disk stars was apparent and was caused by the scalelength of the thick disk being significantly smaller than that of the thin disk. Our rough estimate of the distance for the red clump stars of box B situates many of these stars in the outer disk $\left(R_{\mathrm{GC}} \gtrsim 13 \mathrm{kpc}\right)$. The two disk populations (thin and thick) were detected in our sample of stars in the outer disk. For the nearest red clump stars $(1.2 \leqslant d \leqslant 5.3 \mathrm{kpc})$, we detected three stars with thick-disk properties (stars \#13540, \#26606, and \#32782) and two belonging to the thin disk (stars \#11029 and \#27955). The second most distant star in our sample (star $\# 13964$, with $d \sim 20 \mathrm{kpc}$ ) is also the star with the lowest 
metallicity $([\mathrm{Fe} / \mathrm{H}]=-0.68)$ and has an average abundance of alpha-elements of $[\alpha / \mathrm{Fe}]=0.45 \pm 0.23$. This star by its chemical pattern can be classified as a star of the thick disk or of the Galactic disk-halo transition region. The region of Galactic disk-halo transition is characterized by stars with $-1.20 \leqslant$ $[\mathrm{Fe} / \mathrm{H}] \leqslant 0.55$ and by $\alpha$-poor and $\alpha$-rich stars (Hawkins et al. 2015). Our estimate for the distance of star \#13964 $(d \sim 20 \mathrm{kpc})$, despite its significant uncertainty, may also indicate that this star is located outside the Galaxy. In this case, star \#13964 may have been lost by the Milky Way or belongs to an extragalactic object in the vicinity of the Milky Way. The other two stars situated on the edge of the outer disk (stars \#28064 and \#31364) have a chemical pattern typical of the thin disk $([\alpha / \mathrm{Fe}] \sim 0.0)$. Therefore, our sample, although small, shows that the thin disk is probably dominant in the most extreme regions of the disk, in accordance with the conclusions of Bensby et al. (2011). A study of stars located in the extreme outer regions of the disk can quantify how warped and flared the Galactic disk is as well as how the stellar populations in these regions evolve. Furthermore, the interesting finding of a significant number of stars at the end of the Galactic disk with large estimates of distance from the Galactic plane (like stars \#13964, \#28064, and \#31364) can also reveal a significant mixing between stars from the disk and the halo caused by the warped and flared disk. But what does this interaction between the warped and flared disk and the halo imply for the evolution of stellar populations in this extreme region of the Galaxy? In this region, is the stellar population predominantly $\alpha$-rich or predominantly $\alpha$-poor? And what is the metallicity range? A major difficulty for a reliable study of the structure of extreme outer regions of the disk is an estimated distance that often comes with large uncertainties (as our estimate for star \#13964). Incoming data from the Gaia mission will surely allow more solid distance estimates for many stars and put us in a better position to answer these questions.

The production of $\mathrm{Na}$ in the stellar interior occurs during hydrostatic carbon burning in massive stars (Woosley \& Weaver 1995) and is affected by the $\mathrm{NeNa}$ cycle in the $\mathrm{H}$-burning envelope in intermediate-mass and massive stars (Denisenkov \& Denisenkova 1990). In giant stars, the chemical abundance of $\mathrm{Na}$ is important in investigating the mixing processes occurring in the stellar interior, like the first dredgeup, thermohaline instability, and rotation-induced mixing (Charbonnel \& Lagarde 2010).

The effects of nonlocal thermodynamic equilibrium (NLTE) are considerable with an abundance of $\mathrm{Na}$ (Gratton et al. 1999; Lind et al. 2011) and should be taken into account. In our analysis, we used the corrections of Gratton et al. (1999) to estimate such effects. Table 4 shows the $[\mathrm{Na} / \mathrm{Fe}]$ ratios for six red clump stars. The abundance ratio $[\mathrm{Na} / \mathrm{Fe}]$ for our field red clump stars presents an overabundance of 0.19 to 0.42 . In Figure 5 we see that the $[\mathrm{Na} / \mathrm{Fe}]$ ratios for our sample are overabundant when compared to those of the disk dwarfs from Bensby et al. (2014). The overabundance of the $[\mathrm{Na} / \mathrm{Fe}]$ ratio in giant stars with respect to the ratio in dwarf stars indicates the importance of the chemical mixing phenomena occurring in the stellar interior during the giant phase (Pasquini et al. 2004).

$\mathrm{Al}$ is mainly produced during hydrostatic carbon and neon burning in massive stars (Woosley \& Weaver 1995) and can be affected by the MgAl cycle in H-burning layers at high temperatures (Arnould \& Mowlavi 1995). We observed an

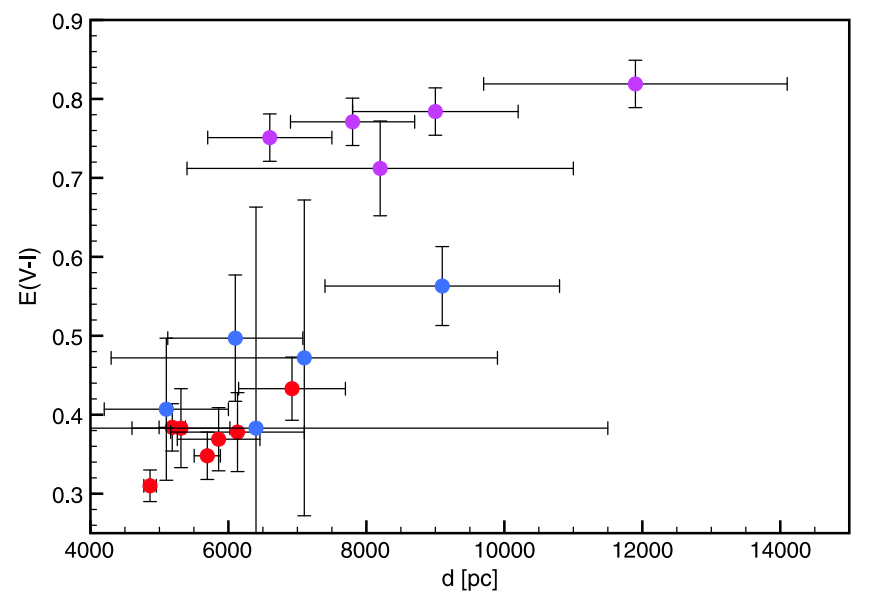

Figure 10. Spectroscopic results of reddening as a function of distance for the targets in boxes $\mathrm{C}$ and $\mathrm{D}$. The blue, violet, and red dots indicate the $\mathrm{C} 1, \mathrm{C} 2$, and $\mathrm{D}$ groups, respectively.

overabundance of $\mathrm{Al}$ with respect to $\mathrm{Fe}$ for our red clump sample, with a range of $0.10 \leqslant[\mathrm{Al} / \mathrm{Fe}] \leqslant 0.59$ (see Table 4). The stars that present the highest values for the $[\mathrm{Al} / \mathrm{Fe}]$ ratio (\#13964, \#26606, and \#32782) are also the stars that have a high overabundance of $\alpha$-elements.

While $\alpha$-elements are overabundant in stellar populations characterized by a fast star formation history, such as the Galactic thick disk, stars in dwarf galaxies are usually $\alpha$ depleted. The reason is that the slow star formation rate in these low-density environments prevents the yields of Type-II supernovae from dominating the pollution of the interstellar medium. In fact, the average $\alpha$-element abundance of Sgr dSph stars in the range $-1 \leqslant[\mathrm{Fe} / \mathrm{H}] \leqslant 0$ is $[\alpha / \mathrm{Fe}] \approx-0.2$ (Sbordone et al. 2007). Similar subsolar $\alpha$-abundances were found by Sbordone et al. (2005) in three field stars, and they were the basis of their claim of an extragalactic origin for their targets. $\mathrm{Na}$ and $\mathrm{Al}$ are also depleted in intermediate-metallicity $\mathrm{Sgr} \mathrm{dSph}$ stars by $\approx-0.3$ and -0.5 dex, likely because the astrophysical sites of their synthesis, massive and intermediate-mass stars, are the same where most of the $\alpha$-elements are produced. In this context, we note that none of our box B stars show abundances that differ from the Galactic trend. The $\alpha$-element, $\mathrm{Na}$, and $\mathrm{Al}$ abundances, along with the Ni content discussed in the previous section, are either close to solar or supersolar for the most metalpoor targets, in full agreement with the general trend of Galactic thin- and thick-disk stars. We therefore conclude that there is no evidence of an extragalactic origin for any of the studied objects.

In the discussion that follows, we put the red clump stars together with the other stars of our sample in the context of the structure of the third Galactic quadrant and try to make connections between the different populations so far analyzed.

\section{Discussion and Conclusions}

In the following we discuss the results of our photometric and spectroscopic analysis and attempt to draw a coherent scenario out of them.

The targets in box D (see Figure 1) belong to a thick, faint MS in the background of the cluster Tombaugh 1 . The observed objects (see Tables 8, 9, and 10) are all F8-G2 spectral type stars with similar reddening, spanning a narrow range of distances $(5-7 \mathrm{kpc}, 5.7 \mathrm{kpc}$ on average). They trace a tight sequence in the CMD. MS stars with these spectral types 
Table 9

Atmospheric Parameters from Spectroscopy of Box C

\begin{tabular}{lcccccc}
\hline \hline ID & $\begin{array}{c}T_{\text {eff }} \\
(\mathrm{K})\end{array}$ & $\begin{array}{c}\log g \\
(\mathrm{dex})\end{array}$ & $\begin{array}{c}v \sin i \\
\left(\mathrm{~km} \mathrm{~s}^{-1}\right)\end{array}$ & $\begin{array}{c}\langle\mathrm{RV}\rangle \\
\left(\mathrm{km} \mathrm{s}^{-1}\right)\end{array}$ & $E(V-I)$ & $\begin{array}{c}d \\
(\mathrm{pc})\end{array}$ \\
\hline $6507^{\mathrm{a}}$ & $14500 \pm 3200$ & $5.5 \pm 1.0$ & 20 & $112 \pm 3$ & $0.68 \pm 0.05$ & $15.68 \pm 0.79$ \\
$8542^{\mathrm{a}}$ & $8100 \pm 4200$ & $4.8 \pm 3.2$ & 120 & $99 \pm 45$ & $0.38 \pm 0.28$ & $14.01 \pm 1.74$ \\
9227 & $9800 \pm 1800$ & $4.2 \pm 0.8$ & 210 & $75 \pm 13$ & $0.47 \pm 0.20$ & $14.21 \pm 0.78$ \\
$12018^{\mathrm{a}}$ & $8700 \pm 800$ & $4.2 \pm 0$ & $0^{\mathrm{a}}$ & $97 \pm 6$ & $0.41 \pm 0.09$ & $13.52 \pm 0.39$ \\
13279 & $13100 \pm 1100$ & $4.4 \pm 0.3$ & 10 & $63 \pm 5$ & $0.78 \pm 0.03$ & $14.63 \pm 0.30$ \\
16940 & $10800 \pm 2400$ & $4.1 \pm 0.3$ & 310 & $69 \pm 11$ & $0.71 \pm 0.06$ & $14.45 \pm 0.75$ \\
24772 & $12000 \pm 1400$ & $4.4 \pm 0.5$ & 60 & $88 \pm 2$ & $0.56 \pm 0.05$ & $14.71 \pm 0.41$ \\
28816 & $9700 \pm 900$ & $3.6 \pm 0.5$ & 70 & $96 \pm 2$ & $0.50 \pm 0.08$ & $13.87 \pm 0.38$ \\
30971 & $11400 \pm 800$ & $4.6 \pm 0.3$ & 90 & $78 \pm 9$ & $0.77 \pm 0.03$ & $14.33 \pm 0.25$ \\
31183 & $14000 \pm 1600$ & $4.4 \pm 0.5$ & 40 & $62 \pm 2$ & $0.82 \pm 0.03$ & $15.22 \pm 0.41$ \\
32089 & $12200 \pm 700$ & $4.3 \pm 0.3$ & 230 & $84 \pm 1$ & $0.75 \pm 0.03$ & $13.98 \pm 0.22$ \\
\hline
\end{tabular}

Note.

${ }^{\mathrm{a}} \mathrm{No} \mathrm{H}_{\beta}$ line.

must be younger than $\approx 9 \mathrm{Gyr}$, and the MS in the CMD continues blueward to even higher temperatures (group in box $\mathrm{C}$, see below). Hence, this MS traces an intermediate-age stellar population, and it cannot be associated with the Galactic halo or thick disk. The distance spread is most likely physical and not only a product of measurement errors, because the stars follow a clear reddening-distance relation, as shown in Figure 10. Two stars slightly depart from this relation, probably due to differential reddening in the FOV. The shape and width of this sequence are comparable with those of the sequences found in the background of NGC 2354 (Carraro et al. 2016) and in the direction of the Canis Major overdensity (Carraro et al. 2008), sequences ascribed to the warped old thin disk, which the line of sight intersects, thus producing the effect of probing a structure confined in distance.

The weighted average $\mathrm{RV}$ is $\sim 107 \mathrm{~km} \mathrm{~s}^{-1}$, much higher than the expectation of a simple Galactic rotation model $\left(\approx 60 \mathrm{~km} \mathrm{~s}^{-1}\right)$, such as that presented by Moni Bidin et al. (2014). However, the model could easily fail at these large distances from the Galactic center and far away from the formal Galactic plane (at latitude $0^{\circ}$ ). The line-of-sight velocity at this Galactic longitude mostly reflects the Galactocentric $U$ component. The RV dispersion, after quadratic subtraction of the mean observational error, is $\sigma_{\mathrm{RV}}=23 \mathrm{~km} \mathrm{~s}^{-1}$. This is very high, because $\sigma_{U} \approx 10 \mathrm{~km} \mathrm{~s}^{-1}$ for the thin disk at the solar position and the dispersion is expected to exponentially decline outward. Hence, this intermediate-age and distant population presents peculiar kinematical properties. A possible explanation for this peculiarity is that this population belongs to the Galactic warp (Momany et al. 2006) and it is also flared (see Carraro et al. 2015 and references therein). In this scenario the kinematics are not easily predictable (see Xu et al. 2015), since the outer disk exhibits several rings and waves, which alter the expected kinematics.

We now continue discussing the stars in box $\mathrm{C}$. These stars form a sequence along the prolongation of the thick MS we just described. In this case they can be interpreted as blue straggler stars of this intermediate-age population. Their color and magnitude can also be compatible with their being thick-disk or halo foreground hot $\mathrm{sdBs}$, although we do not expect so many stars of this type in such a limited volume (Carraro et al. 2015). The spectroscopic data we have analyzed in this work help us to understand better the nature of these stars.

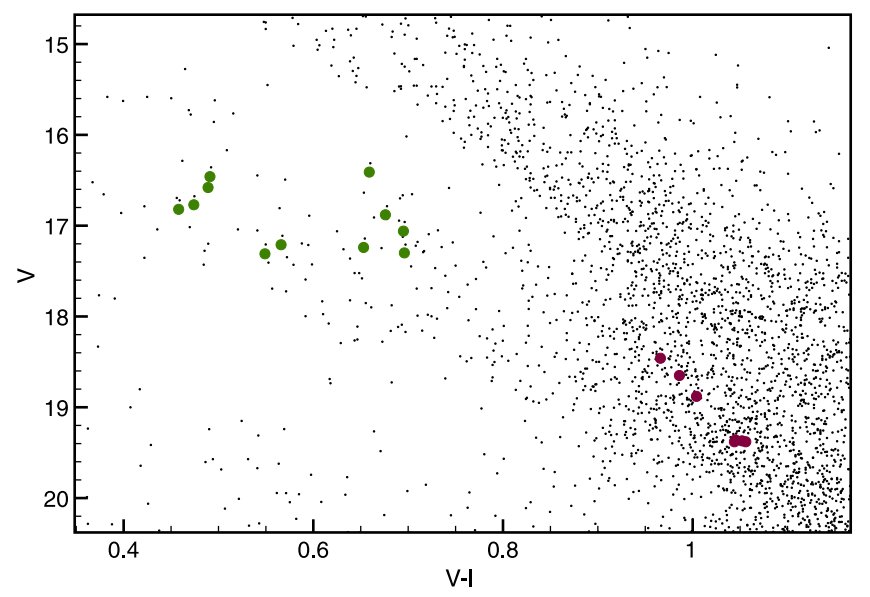

Figure 11. CMD of the field, with box $C$ and box $D$ targets marked with green and red symbols, respectively.

Reading through Table 9 one can infer that these $\mathrm{C}$ group stars are early-type stars, confirming earlier findings (Carraro et al. 2016 and references therein) based on photometry only. Therefore, we remark that the blue plumes routinely found in many different lines of sight in the third quadrant of the Milky Way (Moitinho et al. 2006) are indeed sequences of young stars. Only one of the observed targets is likely an sdB star, as commented in Section 4.3, and it will be excluded from further discussion. For this specific line of sight, a quick glance at the CMDs in Figures 1, 4, and 11 reveals that the stars selected for, and observed with, spectroscopy are clearly separated in the CMD, where there are five stars with $(V-I)<0.57$ (hereafter, the $\mathrm{C} 1$ group) and five objects at $(V-I)>0.65$ (hereafter, C2). The two groups seem to trace two separate sequences. The same dichotomy is found in the spectroscopic results. All C2 objects have $T_{\text {eff }}>10,000 \mathrm{~K}$, while four of the five $\mathrm{C} 1$ stars are cooler than 10,000 K. Thus, C2 stars are on average hotter, despite being redder in the CMD, and in fact they exhibit a much larger reddening $(\overline{E(V-I)}=0.46$ and 0.77 , for the $\mathrm{C} 1$ and $\mathrm{C} 2$ groups, respectively).

The two groups also show different behavior in the distancereddening relation shown in Figure 10. C1 stars are compatible with the distance-reddening relationship defined by the box D targets, and they are distributed in a distance range that largely overlaps that of the box D stars, with a mean value of 
Table 10

Atmospheric Parameters from Spectroscopy of Box D

\begin{tabular}{|c|c|c|c|c|c|}
\hline ID & $\begin{array}{l}T_{\text {eff }} \\
(\mathrm{K})\end{array}$ & $\begin{array}{c}\langle\mathrm{RV}\rangle \\
\left(\mathrm{km} \mathrm{s}^{-1}\right)\end{array}$ & $E(V-I)$ & $(V-M v)_{0}$ & $\begin{array}{c}\mathrm{d} \\
(\mathrm{pc})\end{array}$ \\
\hline 7421 & $5850 \pm 40$ & $76 \pm 8$ & $0.35 \pm 0.03$ & $13.78 \pm 0.09$ & $5700 \pm 200$ \\
\hline 9011 & $6240 \pm 180$ & $101 \pm 12$ & $0.38 \pm 0.05$ & $13.63 \pm 0.29$ & $5300 \pm 700$ \\
\hline 9834 & $6210 \pm 150$ & $74 \pm 3$ & $0.43 \pm 0.04$ & $14.20 \pm 0.24$ & $6900 \pm 800$ \\
\hline 11923 & $\ldots$ & $106 \pm 8$ & $\ldots$ & $\ldots$ & $\ldots$ \\
\hline 19341 & $5990 \pm 200$ & $88 \pm 13$ & $0.38 \pm 0.05$ & $13.94 \pm 0.34$ & $6100 \pm 1000$ \\
\hline 22319 & $5885 \pm 20$ & $125 \pm 6$ & $0.31 \pm 0.02$ & $13.43 \pm 0.05$ & $4860 \pm 100$ \\
\hline 23667 & $6330 \pm 50$ & $139 \pm 7$ & $0.38 \pm 0.03$ & $13.57 \pm 0.10$ & $5200 \pm 200$ \\
\hline 31274 & $5910 \pm 125$ & $135 \pm 2$ & $0.37 \pm 0.04$ & $13.84 \pm 0.22$ & $5900 \pm 600$ \\
\hline 36132 & $\ldots$ & $91 \pm 2$ & $\ldots$ & $\ldots$ & $\ldots$ \\
\hline
\end{tabular}

$d=6.7 \mathrm{kpc}$. C2 stars, on the other hand, are found at nearly constant reddening and on average at a larger distance than box $\mathrm{D}$ and $\mathrm{C} 1$ stars $(d=8.2 \mathrm{kpc}$ on average $)$. In addition, the kinematics of the two C-groups seem different. C1 stars are confined in a narrow range of RVs between 75 and $100 \mathrm{~km} \mathrm{~s}^{-1}$, with a mean value of $91 \mathrm{~km} \mathrm{~s}^{-1}$, similar to that found in box D. The mean RV of the $\mathrm{C} 2$ stars, on the other hand, is $70 \mathrm{~km} \mathrm{~s}^{-1}$, matching within a few $\mathrm{km} \mathrm{s}^{-1}$ the expectations of a simple Galactic rotation model at $d=8 \mathrm{kpc}$. Their RV dispersion is also low, $8.2 \mathrm{~km} \mathrm{~s}^{-1}$, as expected for a thin-disk population.

The observed differences between $\mathrm{C} 1$ and $\mathrm{C} 2$ stars could be partially due to differential reddening. In Figure 12 we show the position of the targets in the Schlegel et al. (1998) reddening map. There is clearly a variation of reddening in the field, and the $\mathrm{C} 1$ (C2) stars tend to be found far from (close to) a local reddening maximum. However, further inspection suggests that this cannot fully explain the observed dichotomy. First, there is a certain degree of mixing in the spatial distribution, with a $\mathrm{C} 2$ target found in a low-reddening area and the object closest to the reddening maximum being a $\mathrm{C} 1$ star. In addition, the reddening variations are small in the Schlegel et al. (1998) map, where they are no more than 0.1 mag in the field under investigation. This is only one third of the difference between the average reddenings of the $\mathrm{C} 1$ and $\mathrm{C} 2$ groups. The variation would even be reduced if corrections to the maps, such as those proposed by Bonifacio et al. (2000), were applied. Differential reddening also cannot explain the different kinematics of the two groups or the distancereddening relation observed for $\mathrm{C} 1$ and $\mathrm{D}$ stars. Moreover, spatial reddening variations alone would cause more reddened stars to be closer, contrary to what is observed, because the targets in each box were selected photometrically at approximately the same magnitude. We conclude that differential reddening may play a role, but it cannot alone explain the observed differences between the $\mathrm{C} 1$ and $\mathrm{C} 2$ groups.

We have to take these differences with some skepticism since they are derived from a handful of stars. From the photometric analysis, in fact we see a continuum of reddening and distance properties, where these two groups, C1 and C2, represent the extremes of these young populations, as seen from the CMD in Figure 11. This discrepancy between the photometric and spectroscopic distribution of reddening and distances in box $\mathrm{C}$ can offer two alternative interpretations: (i) the distribution is broad and continuum-like, as indicated by the photometric results, and the spectroscopic dichotomy is only a spurious effect due to the random selection of a small sample of targets or due to selection biases, or (ii) the underlying distribution is bimodal, as the spectroscopic results suggest, but its nature is lost in the photometric results due to large uncertainties, which blend the two groups into a wider single peak. To investigate this issue in more detail, we compare in Figure 13 the distribution of $E(V-I)$ obtained with the spectroscopic and photometric methods for the same 10 targets of the spectroscopic study. The histogram shows that the bimodal distribution found with the first method is completely lost when the photometrically derived reddening is studied. This result suggests that if the $\mathrm{C} 1$ and $\mathrm{C} 2$ groups represent two distinct stellar populations with different distances and reddening in the same FOV, their presence would have likely been missed in the photometric results. A Kolmogorov-Smirnov test indicates that the probability that the bimodal spectroscopic results are randomly drawn from the photometric broad distribution is only $8 \%$. Hence, the hypothesis that this multimodality is entirely due to the random selection of a small sample of stars cannot be dismissed, but it is very improbable. However, while these tests tend to exclude hypothesis (i) above, they are insufficient to claim the existence of two stellar substructures at different distances in this field, both because the spectroscopic sample is too small and because selection effects unaccounted for in the analysis could have led to an observed sample unevenly distributed in the CMD, thus giving the wrong impression of a bimodal distribution. A more extensive spectroscopic study of a larger sample of stars is required to fix this issue.

This young population is the very same that we found in several other directions in the third Galactic quadrant. It is confined in distance, being at heliocentric distances in the range of 6-9 kpc. Within the uncertainties involved, these stars are most probably tracing a portion of the outer, or Norma-Cygnus, spiral arm. This arm is located well below the formal Galactic plane (at $b=0^{\circ}$ ), because of the warp, and the line of sight to Tombaugh 1 intersects it, in close similarity to the line of sight to the old open cluster Auner 1 (Carraro et al. 2007). As the Norma-Cygnus arm is the outermost arm known of the Milky Way, it is not unexpected to find stars at vastly different distances, because outermost arms are typically wider than inner-disk arms, whose width is typically about $1 \mathrm{kpc}$. Interestingly, the line of sight to Tombaugh 1 does not contain young stars closer to the Sun (at $1.5-2.5 \mathrm{kpc}$ ), which we would expect from the crossing of the Perseus arm, located at about $2 \mathrm{kpc}$ from the Sun (Churchwell et al. 2009). The fact that we miss the Perseus arm means either that the warping of the disk starts to be significant beyond $2-3 \mathrm{kpc}$ or that the Perseus arm is not important in the third Galactic quadrant.

Finally, we comment on the stars located in box B. This box samples red giant stars. According to our results (see Tables 3 


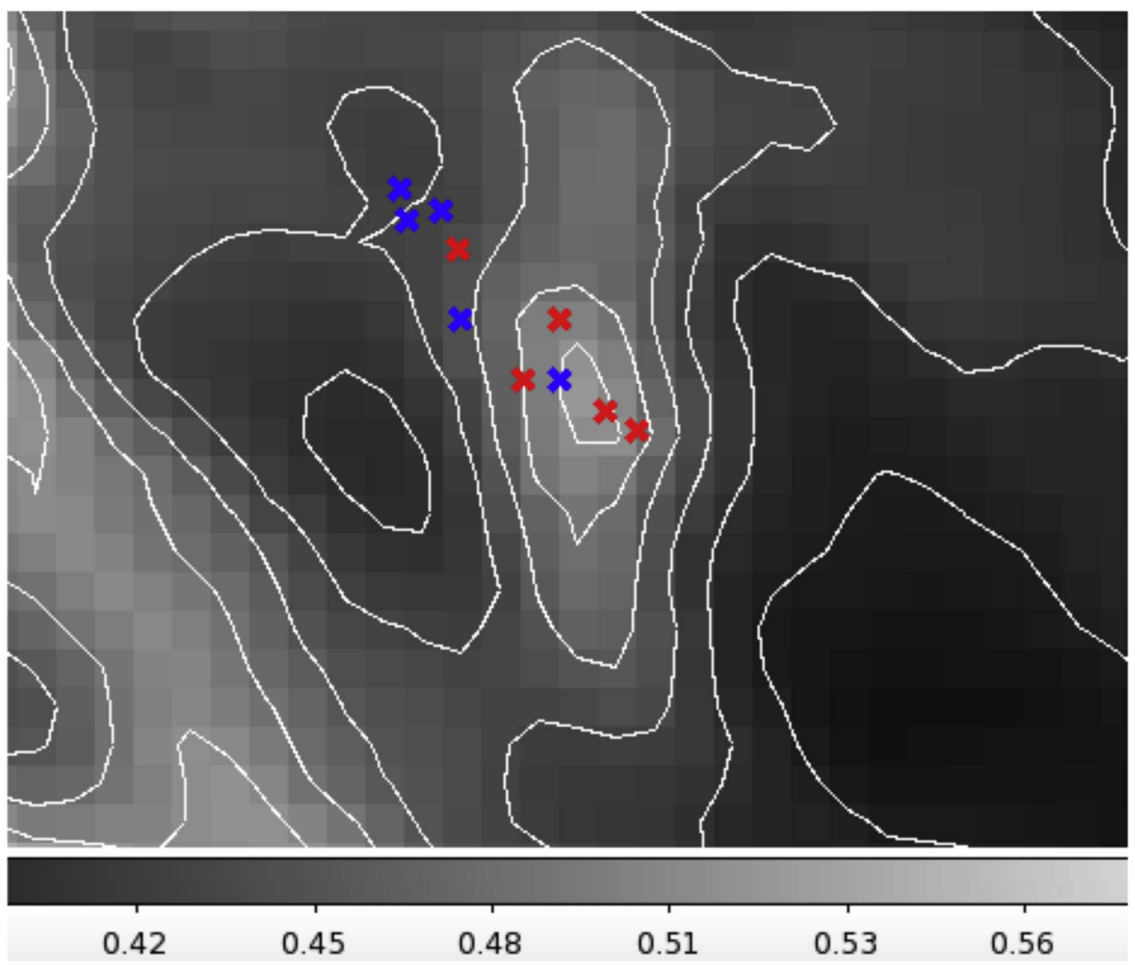

Figure 12. Reddening map from Schlegel et al. (1998) in the region of Tombaugh 1. Blue and red crosses indicate stars from the C1 and C2 subgroups, respectively.

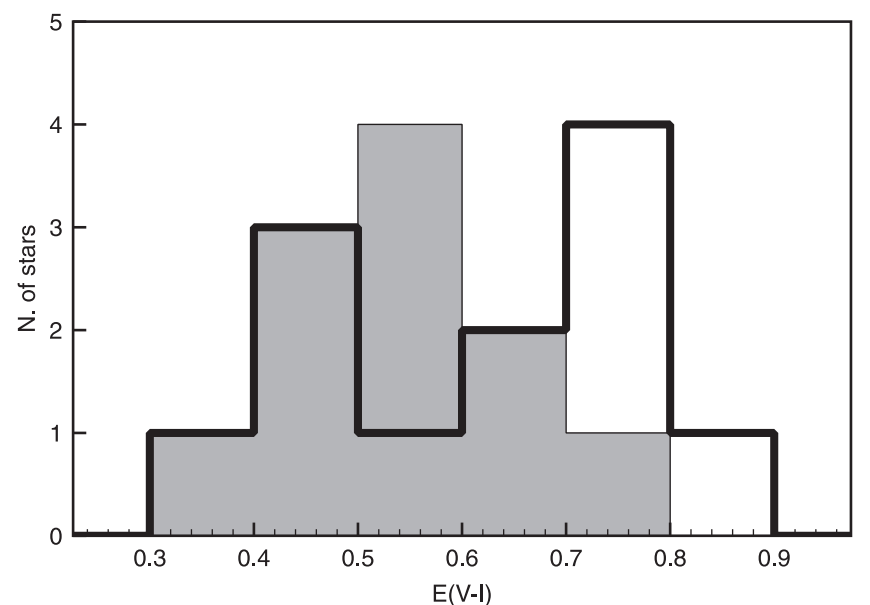

Figure 13. Histogram of the spectroscopic (thick black line) and photometric (shaded gray area) reddening distribution of the box $\mathrm{C}$ stars.

and 4), these stars, which seem to somehow group together in the CMD, are located at different distances and have different metallicities. Thus, the stars of box B have no close relation to the populations of boxes C and D. As discussed in Section 1, the presence of a background MS and a blue plume of hot young stars in the third Galactic quadrant has been interpreted as evidence of the recent accretion of the Canis Major satellite. We argue in Section 5 that we find no evidence of an extragalactic origin for any of our red clump targets. Unfortunately, the lack of a kinematical or spatial link of box B stars with the fainter box C and D groups prevents us from extending the result to these features. However, red clump stars should be present in a complex stellar population, such as that postulated for the Canis Major satellite, and our sample included targets in a wide range of distances. Our results therefore favor the scenario where the peculiar features observed in the CMD are due to a complex mix of Galactic populations rather than to the imprint of an extragalactic system.

We can divide the stars of box B into two field populations. The first is composed of older stars, with ages between $8 \mathrm{Myr}$ and $10 \mathrm{Gyr}$ (belonging to the thick and thin disks, with a slight majority to the thick disk) and distances of $1.2 \leqslant d \leqslant 5.3 \mathrm{kpc}$. The second population is composed of young $(\lesssim 8 \mathrm{Myr})$ background field stars, with high values for distances $(d>12 \mathrm{kpc})$ and slightly similar radial velocities (with a mean $\mathrm{RV}$ of $69 \mathrm{~km} \mathrm{~s}^{-1}$ ). It is important to note that the distances to these background stars of box B have large uncertainties with an average of $\sim 9 \mathrm{kpc}$; these stars are background stars related to the other stars of box B. It is also worth mentioning that the detection of stars with large estimated distances and consequently with large distances from the Galactic plane-such as stars \#13964, \#28064, and \#31364 in box B-and with apparently young age when compared to thick-disk and halo stars is not expected and can reveal an interesting and complex galaxy evolution occurring on the periphery of the warped and flared outer disk with a probable interaction with the Galactic halo. A contribution to this discussion comes from recent Galactic disk simulations revealing that younger populations have increasingly greater scale-lengths at larger distances from the Galactic center (Minchev et al. 2015). This feature reveals the need for further analysis with a large sample of stars in this extreme region of the disk. The Gaia-ESO survey can help us to answer these questions better, since it will enable a reliable determination of the distances of large samples of these stars.

We express our deepest gratitude to Edgardo Costa, who acquired part of the data used for this work. Extensive use was made of the WEBDA database, maintained by E. Paunzen at the 
University of Vienna, Austria (http://www.univie.ac.at/webda). J.V.S.S. acknowledges the support provided by the CNPq/Brazil Science without Borders program (project no. 249122/2013-8). C.M.B. acknowledges support from Fondo Nacional de Investigación Científica y Tecnológica (project no. 1150060).

\section{References}

Alonso, A., Arribas, S., \& Martínez-Roger, C. 1999, A\&AS, 140, 261 Arnould, M., \& Mowlavi, N. 1995, in Proc. 32nd Liege Int. Astrophysical Coll., Stellar Evolution: What Should be Done, ed. A. Noels et al. (Liege: Universite de Liege, Institut d'Astrophysique), 17

Asplund, M., Grevesse, N., Sauval, A. J., \& Scott, P. 2009, ARA\&A, 47, 481 Bellazzini, M., Ibata, R., Monaco, L., et al. 2004, MNRAS, 354, 1263

Bensby, T., Alves-Brito, A., Oey, M. S., Yong, D., \& Meléndez, J. 2011, ApJL, 735, L46

Bensby, T., Feltzing, S., Lundström, I., \& Ilyin, I. 2005, A\&A, 433, 185

Bensby, T., Feltzing, S., \& Oey, M. S. 2014, A\&A, 562, A71

Bergeron, P., Saffer, R. A., \& Liebert, J. 1992, ApJ, 394, 228

Bertelli, G., Girardi, L., Marigo, P., \& Nasi, E. 2008, A\&A, 484, 815

Bertelli, G., Nasi, E., Girardi, L., \& Marigo, P. 2009, A\&A, 508, 355

Bessell, M. S., Castelli, F., \& Plez, B. 1998, A\&A, 333, 231

Bienaymé, O., Famaey, B., Siebert, A., et al. 2014, A\&A, 571, 92

Blackwell, D. E., Booth, A. J., Menon, S. L. R., \& Petford, A. D. 1986, MNRAS, 220, 289

Bonifacio, P., Monai, S., \& Beers, T. C. 2000, AJ, 120, 2065

Carney, B. W., Yong, D., Teixera de Almeida, M. L., \& Seitzer, P. 2005, AJ, 130,1111

Carraro, G., Moitinho, A., \& Vázquez, R. A. 2008, MNRAS, 385, 1597

Carraro, G., Moitinho, A., Zoccali, M., Vazquez, R., \& Baume, G. 2007, AJ, 133,1058

Carraro, G., Monaco, L., \& Villanova, S. 2014a, A\&A, 568, 86

Carraro, G., Seleznev, A. F., Baume, G., \& Turner, D. G. 2016, MNRAS, 455,4031

Carraro, G., Vázquez, R. A., Costa, E., Ahumada, J. A., \& Giorgi, E. E. 2015, AJ, 149,12

Carraro, G., Vázquez, R. A., Costa, E., Perren, G., \& Moitinho, A. 2010, ApJ, 718,683

Carraro, G., Vázquez, R. A., Moitinho, A., \& Baume, G. 2005, ApJL, 630, L153

Carraro, G., Villanova, S., Monaco, L., et al. 2014b, A\&A, 562, 39

Carretta, E., Bragaglia, A., \& Gratton, R. G. 2007, A\&A, 473, 129

Castro, S., Rich, R. M., Grenon, M., Barbuy, B., \& McCarthy, J. K. 1997, AJ, 114,376

Charbonnel, C., \& Lagarde, N. 2010, A\&A, 522, A10

Churchwell, E., Babler, B. L., Meade, M. R., et al. 2009, PASP, 121, 213

Coelho, P., Barbuy, B., Meléndez, J., Schiavon, R. P., \& Castilho, B. V. 2005, A\&A, 443, 735

Denisenkov, P. A., \& Denisenkova, S. N. 1990, SvAL, 16, 275

Depagne, E., Hill, V., Spite, M., et al. 2002, A\&A, 390, 187

Drake, J. J., \& Smith, G. 1991, MNRAS, 250, 89

Dressler, A., Hare, T., Bigelow, B. C., \& Osip, D. J. 2006, Proc. SPIE, 6269, 62690

Edvardsson, B., Andersen, J., Gustafsson, B., et al. 1993, A\&A, 275, 101

Fuhrmann, K., Axer, M., \& Gehren, T. 1994, A\&A, 285, 585

Genovali, K., Lemasle, B., da Silva, R., et al. 2015, A\&A, 580, A17

Gratton, R. G., Carretta, E., Eriksson, K., \& Gustafsson, B. 1999, A\&A, 350, 955

Gratton, R. G., \& Sneden, C. 1988, A\&A, 204, 193

Grevesse, N., \& Sauval, A. J. 1998, SSRv, 85, 161

Hawkins, K., Jofré, P., Masseron, T., \& Gilmore, G. 2015, MNRAS, 453, 758

Horne, K. 1986, PASP, 98, 609

Katime Santrich, O. J., Pereira, C. B., \& de Castro, D. B. 2013, AJ, 146, 39

Keeping, E. S. 1962, Introduction to Statistical Inference (London: Van Nostrand)

Kuijken, K., \& Gilmore, G. 1989, MNRAS, 239, 605

Kurucz, R. 1993, in ATLAS9 Stellar Atmosphere Programs and 2 km/s grid. Kurucz CD-ROM No. 13 (Cambridge, MA: Smithsonian Astrophysical Observatory), 13
Lambert, D. L., Heath, J. E., Lemke, M., \& Drake, J. 1996, ApJS, 103, 183 Landolt, A. U. 1992, AJ, 104, 372

Lee, Y.-W., Joo, S.-J., \& Chung, C. 2015, MNRAS, 453, 3906

Lemasle, B., François, P., Genovali, K., et al. 2013, A\&A, 558, 31

Letarte, B., Hill, V., Jablonka, P., et al. 2006, A\&A, 453, 547

Lind, K., Asplund, M., Barklem, P. S., \& Belyaev, A. K. 2011, A\&A, 528, A103

Magrini, L., Randich, S., Romano, D., et al. 2014, A\&A, 563, 44

Magrini, L., Randich, S., Zoccali, M., et al. 2010, A\&A, 523, 11

Magrini, L., Sestito, P., Randich, S., \& Galli, D. 2009, A\&A, 494, 95

Majaess, D., Carraro, G., Moni Bidin, C., et al. 2013, A\&A, 560, A22

Martin, N. F., Ibata, R. A., Bellazzini, M., et al. 2004, MNRAS, 348, 12

Martin, W. C., Fuhr, J. R., Kelleher, D. E., et al. 2002, NIST Atomic Spectra Database (Gaithersburg, MD: NIST) Version 2.0

Masseron, T., \& Gilmore, G. 2015, MNRAS, 453, 1855

McWilliam, A., \& Rich, R. M. 1994, ApJS, 91, 749

Minchev, I., Martig, M., Streich, D., et al. 2015, ApJL, 804, L9

Mishenina, T., Pignatari, M., Carraro, G., et al. 2015, MNRAS, 446, 3651

Moitinho, A. 2001, A\&A, 370, 436

Moitinho, A., Vázquez, R. A., Carraro, G., et al. 2006, MNRAS, 368, 77

Momany, Y., Zaggia, S., Gilmore, G., et al. 2006, A\&A, 451, 515

Moni Bidin, C., Carraro, G., \& Méndez, R. A. 2012, ApJ, 747, 101

Moni Bidin, C., de la Fuente Marcos, R., de la Fuente Marcos, C., \& Carraro, G. 2010, A\&A, 510, A44

Moni Bidin, C., Majaess, D., Bonatto, C., et al. 2014, A\&A, 561, 119

Moni Bidin, C., Villanova, S., Piotto, G., et al. 2012, A\&A, 547, A109

Moni Bidin, C., Villanova, S., Piotto, G., \& Momany, Y. 2011, A\&A, 528, A 127

Morse, J. A., Mathieu, R. D., \& Levine, S. E. 1991, AJ, 101, 1495

Munari, U., Sordo, R., Castelli, F., \& Zwitter, T. 2005, A\&A, 442, 1127

Napiwotzki, R., Green, P. J., \& Saffer, R. A. 1999, ApJ, 517, 399

Nieva, M. F., \& Przybilla, N. 2007, A\&A, 467, 295

Pasquini, L., Randich, S., Zoccali, M., et al. 2004, A\&A, 424, 951

Patat, F., \& Carraro, G. 2001, MNRAS, 325, 1591

Pereira, C. B., Sales Silva, J. V., Chavero, C., Roig, F., \& Jilinski, E. 2011, A\&A, 533, A51

Preston, G. W., \& Sneden, C. 2001, AJ, 122, 1545

Reddy, A. B. S., Giridhar, S., \& Lambert, D. L. 2013, MNRAS, 431, 3338

Reddy, B. E., Bakker, E. J., \& Hrivnak, B. J. 1999, ApJ, 524, 831

Reddy, B. E., Tomkin, J., Lambert, D. L., \& Allende Prieto, C. 2003, MNRAS, 340, 304

Romero-Gómez, M., Figueras, F., Antoja, T., Abedi, H., \& Aguilar, L. 2015, MNRAS, 447, 218

Saffer, R. A., Bergeron, P., Koester, D., \& Liebert, J. 1994, ApJ, 432, 351

Sales Silva, J. V., Carraro, G., Anthony-Twarog, B. J., et al. 2016, AJ, 151, 6

Sbordone, L., Bonifacio, P., Buonanno, R., et al. 2007, A\&A, 465, 815

Sbordone, L., Bonifacio, P., Marconi, G., Zaggia, S., \& Buonanno, R. 2005, A\&A, 430, L13

Sbordone, L., Monaco, L., Moni Bidin, C., et al. 2015, A\&A, 579, 104

Schlegel, D. J., Finkbeiner, D. P., \& Davis, M. 1998, ApJ, 500, 525

Sneden, C. 1973, ApJ, 184, 839

Sneden, C., Ivans, I. I., \& Fulbright, J. P. 2004, in Carnegie Observatories Centennial Symp., Origin and Evolution of the Elements, ed. A. McWilliam \& M. Rauch (Cambridge: Cambridge Univ. Press), 170

Stetson, P. B. 1987, PASP, 99, 191

Tonry, J., \& Davis, M. 1979, AJ, 84, 1511

Turner, D. G. 1976, AJ, 81, 97

Turner, D. G. 1979, PASP, 91, 642

Turner, D. G. 1983, JRASC, 77, 31

Turner, D. G., MacLellan, R. F., Henden, A. A., \& Bernikov, L. N. 2011a, RMxAA, 47, 345

van Dokkum, P. G. 2001, PASP, 113, 1420

Vazquez, R. A., May, J., Carraro, G., et al. 2008, ApJ, 672, 930

Wan, J.-C., Liu, C., Deng, L.-C., et al. 2015, RAA, 15, 1166

Wiese, W. L., Smith, M. W., \& Miles, B. M. 1969, NSRDS-NBS (Washington, DC: US Department of Commerce, National Bureau of Standards)

Woosley, S. E., \& Weaver, T. A. 1995, ApJS, 101, 181

Xu, Y., Newberg, H. J., Carlin, J. L., et al. 2015, ApJ, 801, 105

Yong, D., Carney, B. W., Teixera de Almeida, M. L., \& Pohl, B. L. 2006, AJ, 131,2256 